\title{
Interactive Cellular and Cordless Video Telephony: State-of-the-Art System Design Principles and Expected Performance
}

\author{
LAJOS HANZO, SENIOR MEMBER, IEEE, PETER CHERRIMAN, ASSOCIATE MEMBER, IEEE, AND \\ EE-LIN KUAN
}

\begin{abstract}
Second-generation $(2 G)$ mobile radio standards have not been designed with video communications in mind, although the employment of error-resilient, constant-bit-rate proprietary video codecs over these systems is realistic. The third-generation $(3 G)$ systems are capable of providing higher rates and better communications integrity in support of video applications. This paper advocates the employment of burst-by-burst adaptive transceivers, which are capable of accommodating the time-variant channel quality fluctuation of wireless channels. This paper is concluded with a range of performance figures and system design guidelines for wireless systems.
\end{abstract}

Keywords-Burst-by-burst adaptive video transmission, H.263 codec, joint detection CDMA, MPEG4 codec, power control, $3 G$ systems, $2 G$ systems, video, video over cordless telephones, video telephony, wireless video, wireless video communications.

\section{STATE-OF-THE-ART SyStem COMPONENT DEVELOPMENTS}

\section{A. Introduction and Outline}

The subject of mobile radio communications has reached a state of maturity over the past two decades, as indicated by the excellent monographs by Jakes [1], Lee [2]-[4], Parsons and Gardiner [5], Parsons [6], Pahlavan and Levesque [7], Feher [8], Goodman [9], Prasad [10], Rappaport [11], Garg and Wilkes [12], Gibbson [13], Glisic and Vucetic [14], Verdu [16], and Meyr et al. [15]. Recent third-generation (3G) mobile research has been strongly motivated by the seminal contributions of Viterbi [17], while a range of European contributions were summarized in [18]. These advances have also been featured in the state-of-the-art collection of high-quality contributions edited by Glisic and Leppänen [19] and in various magazine special issues [20]-[24] and other review articles [25]. A range of further narrow-band multimedia system components were reviewed in [26], [27].

Manuscript received September 1, 1998; revised June 12, 2000.

The authors are with the Department of Engineering and Computer Science, University of Southampton, Southampton SO17 1BJ, U.K.

Publisher Item Identifier S 0018-9219(00)08966-0.
This contribution attempts to summarize a range of recent advances in the field of bandwidth-efficient wireless video communications. In this section, we commence our discourse with a brief overview of the wireless scene and, in particular, by considering the video transmission capabilities of the existing and future wireless systems. The geographical variation of the cellular channel capacity is characterized by Section II, as a motivation for invoking burst-by-burst adaptive transceivers [27]-[29]. The nature of co-channel interference (CCI) - which is the most dominant channel impairment in wireless systems - is the topic of Section III, where it is shown that the geographic variation of CCI justifies the employment of multimode transceivers, which are discussed in Section IV. Low-rate video compression aspects are reviewed in Section V, and the performance of the ITU H.263 codec is analyzed in some depth. Our discussions in Sections VI and VII are focused on the performance of reconfigurable video transceivers. Specifically, in Section VI, no power control is used-which is typically the case in cordless telephones, such as DECT and CT2 - while in Section VII, power control is employed, which is characteristic of cellular systems, such as GSM [30], IS-136, IS-95, etc. Section VIII proposes a near-instantaneously adaptive or burst-by-burst adaptive code-division multiple-access (CDMA) scheme, which can be invoked in the context of the forthcoming $3 \mathrm{G}$ systems, in order to enhance their performance. Lastly, our discussions are concluded in Section IX with a range of system design guidelines. Let us now commence our overview of the range of existing and forthcoming wireless systems and their video transmission capabilities.

\section{B. Second-Generation (2G) Wireless Systems}

The existing $2 \mathrm{G}$ wireless systems now constitute a mature technology. Although they have not been designed with video communications in mind, with the advent of the specially designed error-resilient, fixed-rate video codecs [27]-[35] proposed by Streit et al., it is nonetheless realistic to provide videophone services over these low-rate schemes. For low-latency interactive videophony these systems have 
Table 1

Speech Coding Rates of 2G Mobile Systems

\begin{tabular}{|c|c|c|c|c|c|c|c|c|c|}
\hline \multirow[b]{2}{*}{ System } & \multicolumn{5}{|c|}{ Cellular Systems } & \multicolumn{4}{|c|}{ Cordless Systems } \\
\hline & GSM [39] & $\begin{array}{l}\text { DCS- } \\
1800 \\
\end{array}$ & $\begin{array}{l}\text { IS-95A/B }[41,70] \\
\text { CDMA }[42,43,44]\end{array}$ & IS-54/IS-136 & $\mathrm{JDC}[45]$ & CT2 [46] & DECT [13] & PHS [47] & $\operatorname{PACS}[48]$ \\
\hline Origin & Europe & Europe & USA & $\overline{\text { USA }}$ & Japan & UK. & Europe & Japan & USA \\
\hline Voice+FEC Rate(kbit/s) & 22.8 & 22.8 & 8/Var. & 11.2 & & 32 & & & 32 \\
\hline Unprotected Voice Rate(kbit/s) & 13 & 13 & $1.2-9.6$ & $7.95 / 7.4$ & 6.7 & 32 & 32 & 32 & 32 \\
\hline $\begin{array}{l}\text { Speech Forward Error } \\
\text { Correction Coding (FEC) }\end{array}$ & $\begin{array}{l}\text { Conv. } \\
(2,1,5)\end{array}$ & $\begin{array}{l}\text { Conv. } \\
(2,1,5)\end{array}$ & $\begin{array}{l}\text { Conv. } \\
\text { UL: }(2,1,9) \\
\text { DL: } R=1 / 3\end{array}$ & $\begin{array}{l}\text { Conv. } \\
(2,1,5)\end{array}$ & $\begin{array}{l}\text { Conv. } \\
R=9 / 17\end{array}$ & No & No & CRC & CRC \\
\hline $\begin{array}{l}\text { DCS-1800: GSM-like European s } \\
\text { IS-95: American CDMA system } \\
\text { PHS: Japanese Personal Handyp } \\
\text { JDC: Japanese Digital Cellular } \\
\text { CDMA: Code Division Multiple } \\
\text { Conv.: Convolutional coding wit }\end{array}$ & $\begin{array}{l}\text { stem in the } \\
\text { one System } \\
\text { ccess } \\
\text { a rate of } R\end{array}$ & $1800 \mathrm{MH}$ & band & $\begin{array}{l}\text { IS-54: Americ } \\
\text { CT2: British } \\
\text { PACS: Persor } \\
\text { GSM: Global } \\
\text { DECT: Digit: } \\
\text { UL: Up-link, }\end{array}$ & $\begin{array}{l}\text { an Digital } \\
\text { Cordless } \mathrm{T} \\
\text { al Access } \\
\text { System of } \\
\text { 1 European } \\
\text { DL: Down-1 }\end{array}$ & $\begin{array}{l}\text { dvanced M } \\
\text { ephone Sys } \\
\text { mmunicat } \\
\text { obile Com } \\
\text { Cordless Tt } \\
\text { k }\end{array}$ & $\begin{array}{l}\text { obile Phone S } \\
\text { ons System } \\
\text { nunications } \\
\text { lephone }\end{array}$ & ystem (DA & APS) \\
\hline
\end{tabular}

to use an additional speech channel, ${ }^{1}$ since the typical mobile radio data channels that are available at the time of writing typically exhibit a higher delay than the speech channel due to the increased interleaving delay required for maintaining a higher error resilience. Therefore, the existing data channels are typically less amenable to interactive real-time videophony. The associated bit rates of the $2 \mathrm{G}$ speech systems - which would be, therefore, also applicable to the video channel-are summarized in Table 1, along with their acronyms and an associated background reading concerning the various other parameters of these systems. We note, furthermore, that with the advent of the emerging so-called general packet radio system known as GPRS [37] and the high-speed circuit switched data (HSCSD) [36] channel, the employment of multiple time slots per user becomes possible, which renders the system more amenable to video telephony [27], [38].

Let us consider first the $2 \mathrm{G}$ cellular systems represented, for example, by the global system of mobile communications known as GSM [39], [40] and its $1800-\mathrm{MHz}$ carrier-frequency "up-converted" version known as the DCS 1800 system, the Pan-American IS-95 (CDMA) scheme [41], the Pan-American IS-54/IS-136 [42]-[44], and the Japanese digital cellular (JDC) arrangement [45] summarized in the left half of Table 1. These large-cell cellular systems have a speech rate of less than or equal to $13 \mathrm{~kb} / \mathrm{s}$. By contrast, the cordless telephone (CT) schemes summarized in the right half of Table 1, such as the CT2 [46], the digital European cordless telephone (DECT) [13], the Japanese personal handyphone system [47] (PHS), and the American PACS [48] system, all employ $32-\mathrm{kb} / \mathrm{s}$ speech codecs and, hence, are more amenable to video communications at this increased rate.

Apart from their bit rate differences, a further important difference between cellular and cordless systems is that the low-complexity cordless systems typically refrain from using power control, while the more complex cellular systems often operate over hostile channels and usually have quite elaborate power-control schemes. Hence, we will provide expected video performance results for both types of systems. Specifically, in Section VI, no power control is invoked, while in Section VII, power control is employed.

${ }^{1}$ Provided that this is affordable in capacity terms.

\section{Video Aspects}

The philosophy of the fixed but programmable-rate proprietary video codecs and systems presented by Streit $e t$ $a l$. in [27]-[35] was that irrespective of the video motion activity experienced, the specially designed proprietary video codecs generated a constant number of bits per video frame. This constant bit rate was achieved not by the conventional technique of incorporating bit-rate fluctuation smoothing buffering, which would have a feedback to the bit-rate control scheme of the codec and, hence, would increase the delay of the codec. Instead, the generated bit rate was kept constant by configuring the codecs to identify a fixed number of perceptually most important blocks and quantizing them using a constant number of bits per video frame. Furthermore, the philosophy of [27]-[35] was that in order to cope with the high bit error rate (BER) of wireless channels, in some of the codecs it was necessary to refrain from using high-compression variable bit rate so-called run-length coding techniques, hence, accepting some coding inefficiency. This, however, allowed us to generate a video bit stream exhibiting increased error resilience. For example, for videophony over the GSM system at $13 \mathrm{~kb} / \mathrm{s}$ and assuming a scanning rate of 10 video frames/s, 1300 bits per video frame have to be generated. ${ }^{2}$ Additionally, two families of codecs were designed by Streit et al. [27]-[35], one refraining from using error-sensitive run-length coding techniques and exhibiting the highest possible error resilience, and another aiming at the highest possible compression ratio. This fixed-rate approach had the advantage of requiring no bit-rate-smoothing buffering and, hence, exhibiting no objectionable video latency or delay, which would jeopardize lip-synchronization between the voice and video signals [27]-[35].

The fixed bit rate of the above proprietary video codecs is in contrast to the variable rate of the existing standard video codecs, such as the Motion Pictures Expert Group codecs known as MPEG1 [49], MPEG2 [50], and MPEG4 [51], [52], [169]-[171] or the H.261 and H.263 codecs [27], [53] standardized by the International Telecommunications Union (ITU). A distinctive feature of the latter standard codecs is, namely, that the time-variant video motion activity

${ }^{2} \mathrm{~A}$ range of video sequences encoded at various constant bit rates between 6.7 and $13 \mathrm{~kb} / \mathrm{s}$ can be viewed at http://www-mobile.ecs.soton.ac.uk. 
and the variable-length coding techniques employed result in a time-variant bit rate fluctuation due to their effort to maintain a near-constant perceptual video quality.

In this paper, we summarize a range of techniques, which can be invoked in order to render the family of variablelength coded, highly bandwidth-efficient, but potentially error-sensitive class of standard video codecs-such as the H.261, H.263, MPEG1, MPEG2, and MPEG4 arrangements-amenable to error-resilient, low-latency interactive wireless multimode videophony. ${ }^{3}$ A near-constant bit rate was generated upon reencoding the video sequence a number of times, until, for example, a so-called macro-block (MB) constituted by four $8 \times 8$-pixel video blocks generated the required number of bits. This was achieved upon invoking different quantizers from the legitimate set of quantizers in the H.263 scheme [142]. These measures will be highlighted in more depth during our further discourse.

\section{Transmission Issues}

Focusing our attention on transmission issues, in Europe, the wireless communications community opted for employing CDMA [8], [10], [14] combined with time-division multiple access (TDMA) and with time-division duplexing (TDD) in the $3 \mathrm{G}$ personal communications network (PCN) referred to as the universal mobile telecommunications system (UMTS) [68]. The corresponding personal communications system (PCS) emerging from the currently operational IS-95 system is the so-called cdma2000 arrangement in North America, while the ITU completed the so-called intelligent mobile terminal in the year 2000 recommendation, which is termed as the IMT2000 standard [68].

\section{E. 3G Wireless Systems}

1) UMTS and IMT-2000 [60]-[68]: As mentioned above, the radio access scheme of both UMTS and IMT-2000 is wide-band CDMA, and both frequency-division duplex (FDD) and TDD operations are supported. In FDD systems, the uplink (UL) and downlink (DL) signals are transmitted using different carrier frequencies in paired duplex bands. By contrast, the uplink and downlink messages in the TDD mode are transmitted using different time slots. An advantage of the TDD mode is that it can be readily used in applications, where the bit-rate requirements are unbalanced between the two directions, such as in video on demand. Specifically, in such an asymmetric-rate application supported by the FDD mode, either the uplink or the downlink frequency slot would be unexploited, while in the TDD mode, the slots can be assigned on a more flexible basis, potentially doubling the capacity of the link by allocating all time slots in one direction.

The parameters of the FDD and TDD modes are mutually compatible, facilitating the implementation of a dual-mode handset capable of accessing the services offered by both

\footnotetext{
${ }^{3}$ We note here that in the wireless community, the terminology "multimode" often implies actually "multistandard" operation, such as, e.g., GSM/DECT or DAMPS/IS95. However, in this paper, we use the multimode expression in order to indicate that the modem can be configured in a number of operational modes.
}

Table 2

UTRA/IMT-2000 System Parameters (From [68])

\begin{tabular}{l|l}
\hline Radio Access Technology & $\begin{array}{l}\text { FDD : DS-CDMA } \\
\text { TDD : TDMA/CDMA }\end{array}$ \\
\hline Operating environments & Indoor/Outdoor to indoor/Vehicular \\
\hline Chip rate (Mcps) & 3.84 \\
\hline Channel bandwidth (MHz) & 5 \\
\hline Nyquist roll-off factor & 0.22 \\
\hline Duplex modes & FDD and TDD \\
\hline Channel bit rates (kbps) & FDD (UL) : 15/30/60/120/240/480/960 \\
& FDD (DL) : 15/30/60/120/240/480/960/1920 \\
& TDD (UL) ${ }^{\dagger}:$ variable, from 366 to 6624 \\
\hline Tx. Frame length & TDD (DL) $: 366 / 414 / 5856 / 6624$ \\
\hline Spreading factor & FDD (UL) : variable, 4 to 256 \\
& FDD (DL) : variable, 4 to 512 \\
& TDD (UL) : variable, 1 to 16 \\
\hline Detection scheme & TDD (DL) : 1, 16 \\
\hline Inter-cell operation & Coherent with time-multiplexed pilot symbols \\
& Coherent with common pilot channel \\
\hline Power control & FDD : Asynchronous \\
& TDD : Synchronous \\
\hline Transmit power range & Inner-loop \\
\hline Handover & Open loop (TDD UL) \\
\hline Channel bit rate per timeslot & Soft handover \\
\hline & Inter-frequency handover \\
\hline
\end{tabular}

Table 3

Parameters of the cdma2000 System (From [68])

\begin{tabular}{l|l}
\hline Radio Access Technology & DS-CDMA, Multicarrier CDMA \\
\hline Operating environments & Indoor/Outdoor to indoor/Vehicular \\
\hline Chip rate (Mcps) & $1.2288 / 3.6864 / 7.3728 / 11.0592 / 14.7456$ \\
\hline Channel bandwidth $(\mathrm{MHz})$ & $1.25 / 3.75 / 7.5 / 11.25 / 15$ \\
\hline Duplex modes & FDD and TDD \\
\hline Tx. Frame length & 5 and $20 \mathrm{~ms}$ \\
\hline Spreading factor & variable, 4 to 256 \\
\hline Detection scheme & Coherent with common pilot channel \\
\hline Inter-cell operation & FDD : Synchronous \\
& TDD : Synchronous \\
\hline Power control & Open- and closed-loop \\
\hline Handover & Soft-handover \\
& Inter-frequency handover \\
\hline
\end{tabular}

FDD and TDD operators. We note furthermore that recent research advocates the TDD mode quite strongly in the context of burst-by-burst adaptive CDMA modems [69], since the uplink-downlink channel quality similarity can be advantageously exploited in order to adjust the modem parameters, such as the spreading factor or the number of bits per symbol on a burst-by-burst basis according to the instantaneous channel quality. This allows the system to more efficiently exploit the time variant wireless channel capacity by invoking a more robust modulation mode under hostile instantaneous channel conditions, whilst transmitting a higher number of bits per symbol during favorable channel conditions.

Table 2 summarizes the parameters of the so-called terrestrial radio access (UTRA) scheme of the UMTS and those of the IMT-2000 system. The most important ones from a video communications perspective are the bit rates supported, which can be found in row seven of Table 2 . As expected from a flexible $3 \mathrm{G}$ scheme, there are a wide variety of possible video bit rates, ranging from $16 \mathrm{~kb} / \mathrm{s}$ to $4.096 \mathrm{Mb} / \mathrm{s}$. This allows the systems to support a wide range of video resolutions, an issue that will be discussed in the context of Sections V-B and V-C. In summary, the UTRA/IMT-2000 systems are significantly more amenable 
to video communications due to their higher bit rates than the $2 \mathrm{G}$ systems of Table 1 .

Both the UTRA and the IMT-2000 systems exhibit a basic chip rate of $4.096 \mathrm{Mc} / \mathrm{s}$, requiring a bandwidth of $5 \mathrm{MHz}$, when using root-raised cosine Nyquist pulse shaping filters [28] with an excess bandwidth of 0.22. IMT-2000 has an additional lower chip rate of $1.024 \mathrm{Mc} / \mathrm{s}$, corresponding to a bandwidth of $1.25 \mathrm{MHz}$. Increased chip rates of 8.192 and $16.384 \mathrm{Mc} / \mathrm{s}$ are also specified in order to cater for much higher user bit rates $(>2 \mathrm{Mb} / \mathrm{s})$.

2) The cdma2000 System [70]-[72]: The current 2G CDMA-based mobile radio systems standardized by the Telecommunications Industry Association (TIA) in the United States are IS-95-A and IS-95-B [70], [72]. As seen in Table 1, the radio access technology of both systems is based on narrow-band DS-CDMA with a chip rate of 1.2288 $\mathrm{Mc} / \mathrm{s}$, which gives a bandwidth of $1.25 \mathrm{MHz}$. IS-95-A was introduced in 1995, supporting a maximum bit rate of $14.4 \mathrm{~kb} / \mathrm{s}$ [70], [72]. An enhancement to the IS-95-A standards, known as IS-95-B, was launched in 1998 with the objective of supporting data rates up to $115.2 \mathrm{~kb} / \mathrm{s}$ [74], [18], [62], while essentially retaining the physical layer of the original IS-95-A system. In order to meet the $3 \mathrm{G}$ mobile radio system requirements, recently the TIA has ratified cdma2000 as the Pan-American 3G mobile radio system. However, the frequency bands allocated for the $3 \mathrm{G}$ mobile radio systems during the World Administrative Radio Conference (WARC'92) were 1885-2025 MHz and 2110-2200 $\mathrm{MHz}$, which have already been partially assigned to the IS-95 system in the frequency bands of $1850-1910 \mathrm{MHz}$ and 1930-1990 MHz. Hence, the cdma2000 system was designed such that it can be overlaid on IS-95, and it is backward compatible with IS-95.

The parameters of the cdma2000 system are summarized in Table 3, which has a basic chip rate of $3.6864 \mathrm{Mc} / \mathrm{s}$ that is $3 \times$ the $1.2288 \mathrm{Mc} / \mathrm{s}$ of the IS-95 system. Hence, the bandwidth was also tripled to $3.75 \mathrm{MHz}$. Due to the integer relationship between the associated chip rates, the existing IS-95 infrastructure is capable of supporting the operation of the forthcoming cdma2000 system. Higher chip rates of $N \times$ $1.2288 \mathrm{Mc} / \mathrm{s}, N=6,9,12$ are also supported in cdma2000, in order to facilitate transmissions at substantially increased bit rates, supporting, for example, high-rate file transfer and high-resolution video communications. The parameter $N$ determines the channel coding rate and the channel bit rate supported. In order to support, for example, high-bit-rate video communications, $N>1$ has to be used, and in this scenario two different modulation modes may be invoked. In the direct-spread modulation mode, the symbols are spread according to the chip rate and transmitted using a single carrier, giving a bandwidth of $N \times 1.25 \mathrm{MHz}$. This method is used on both the uplink and downlink. In the multicarrier (MC) modulation mode, the symbols to be transmitted are demultiplexed into separate signals, each of which is then spread at a chip rate of $1.2288 \mathrm{Mc} / \mathrm{s}$. $N$ different carrier frequencies are used to transmit these spread signals, each of which has a bandwidth of $1.25 \mathrm{MHz}$. This method is used for the downlink only, since in this case, transmit diversity can be achieved by transmitting the different carrier frequencies over spatially separated antennas. Similarly to UTRA and IMT-2000, cdma2000 also supports TDD operation, which potentially doubles the capacity of the link, when there is a high proportion of asymmetric-rate traffic. This system was also designed for supporting dual-mode FDD/TDD operation, in order to be able to benefit from the services provided by both FDD and TDD operators.

In cdma2000 there are two types of physical channels, namely, dedicated physical channels (DPHCHs) and common physical channels (CPHCHs). As suggested by the terminology, DPHCHs are used to communicate between the base station (BS) and a single mobile station (MS), while $\mathrm{CPHCHs}$ carry information between the base station and several mobile stations. Furthermore, there are two different DPHCHs, namely, the fundamental channel (FCH) and the supplemental channel $(\mathrm{SCH})$, in order to support multiple simultaneous services, such as simultaneous conversations with several parties, file transfers, web browsing, etc. Each channel carries a different type of service and is coded and interleaved independently. However, the number of FCHs is limited to one in any connection, which can be supported by several SCHs.

There are two possible transmission frame durations, namely, 20 and $5 \mathrm{~ms}$. For an FCH transmitted in a 20-ms frame, two sets of uncoded data rates, denoted as Rate Set 1 (RS1) and Rate Set 2 (RS2), are supported. The data rates in $\mathrm{RS} 1$ and RS2 are 9.6/4.8/2.7/1.5 kb/s and 14.4/7.2/3.6/1.8 $\mathrm{kb} / \mathrm{s}$, respectively. Regardless of the uncoded data rates, the channel coded data rate is 19.2 and $38.4 \mathrm{~kb} / \mathrm{s}$ for RS1 and RS2, respectively, when the rate-control parameter is $N=1$. The 5 -ms transmission frame only supports one data rate, which is $9.6 \mathrm{~kb} / \mathrm{s}$. The $\mathrm{SCH}$ is capable of transmitting higher data rates than the $\mathrm{FCH}$, ranging from $1.5 \mathrm{~kb} / \mathrm{s}$ for $N=1$ to as high as $2073.6 \mathrm{~kb} / \mathrm{s}$, when $N=12$. Blind rate detection [73] is used for SCHs not exceeding $14.4 \mathrm{~kb} / \mathrm{s}$, while rate information is explicitly provided for higher data rates. The dedicated control physical channel has a fixed uncoded data rate of 9.6 $\mathrm{kb} / \mathrm{s}$ on both 5 - and 20-ms transmission frames. This control channel rate is about an order of magnitude higher than that of the IS-95 system, and, hence, supports a substantially enhanced system control, which can be advantageously exploited in wireless video communications. Given the above-mentioned bit rates and control channel rates, all $3 \mathrm{G}$ systems are amenable to interactive video communications using a wide range of video resolutions and bit rates-provided that the system design guidelines summarized during our further discourse can be followed.

In summary, 3G systems are expected to exhibit a high flexibility in terms of bit rates, mobility, and services. Ideally, they are backward compatible with $2 \mathrm{G}$ systems while having advanced adaptive features, some of which constitute the topic of our further discourse in this contribution.

Following the above brief overview of the $3 \mathrm{G}$ mobile system standardization activities and system components, let us now briefly elaborate on the capacity of wireless channels in Section II, which, again, provides the justification for using intelligent multimode transceivers. 


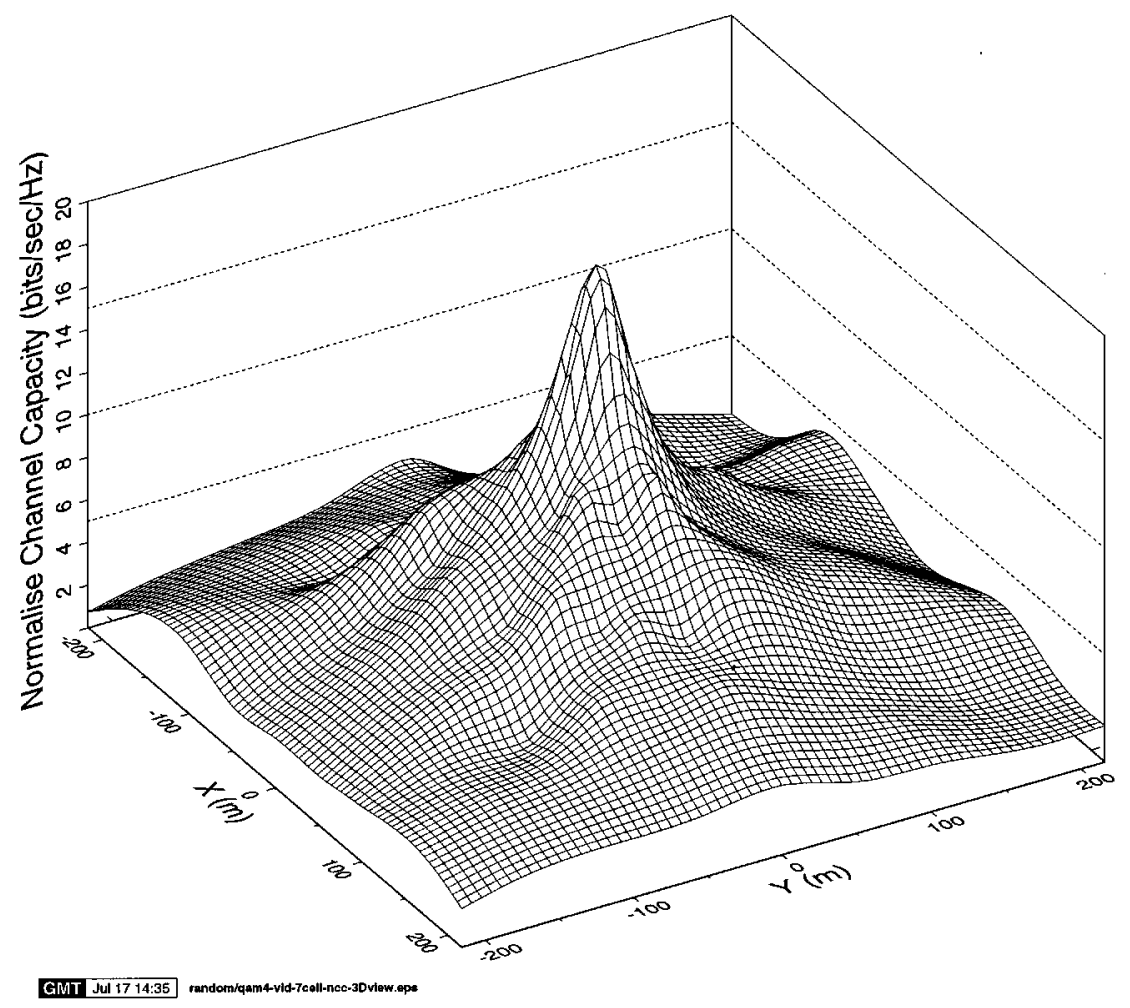

Fig. 1. Simulated normalized channel capacity profile of a hexagonal cell without power control, employing a reuse factor of seven - corresponding to six first-tier interferers-a path loss exponent of 3.5, slow-fading frequency of $1 \mathrm{~Hz}$, standard deviation of $6 \mathrm{~dB}$, and six stationary 4-QAM uplink video interferers at random positions within cell boundaries (from [142]).

\section{Cellular Channel Capacity Without Power CONTROL}

In this section, we set out to make it plausible that the channel capacity across the geographic area of a cell varies over a wide range, suggesting that an intelligent multimode video transceiver has potential advantages over nonreconfigurable transceivers, capable of better exploiting the nonuniform geographic distribution of the channel capacity offered by the system. Even in the power-controlled cellular-rather than cordless-systems, it is beneficial to configure the transceiver in a robust modulation mode near the fringes of the cell, where more hostile channel conditions prevail. Lastly, burst-by-burst adaptive transceivers [27]-[29] are capable of adapting to the short-term time-variant channel quality fluctuations of wireless channels expressed in terms of the BER or transmission burst error rate, irrespective of whether their source was the time-variant co-channel and/or intersymbol interference, slow and/or fast fading, etc. The range of already operational adaptive transceivers was reviewed in the excellent contribution by Nanda et al. [75].

More explicitly, in most mobile radio systems, the channel exhibits Rayleigh fast fading, aggravated by typically lognormally distributed shadowing or slow fading, resulting in a time-variant channel capacity. Lee [76] derived an estimate of the channel capacity in Rayleigh fading environments as a function of the channel signal-to-noise ratio (SNR).

In a cellular frequency reuse structure, the effect of co-channel interference must be included in the channel capacity estimate. Hence, the channel capacity formula proposed by Lee [76] must be modified by replacing the SNR with the signal-to-interference-plus-noise ratio (SINR), provided that the interference is due to a sufficiently high number of interferers and, hence, can be considered near-Gaussian due to the central limit theorem. When this condition is not met, the above approximation will result in optimistic channel capacity estimates. The adjacent channel interference in these elaborations was neglected since in all of our simulation experiments, a Nyquist excess bandwidth of $50 \%$ was used, which provides a sufficiently wide transition band for the receiver filters for this condition to be justified [28]. The SINR is defined as

$$
\gamma=\frac{S}{I+N}
$$

where

$S$ received signal power;

$I$ received interference power;

$N$ additive white Gaussian noise power within the bandwidth of the channel.

In a noise-limited radio system without power control, one would expect the SINR to reduce with distance from the transmitter when using an omnidirectional aerial. However, in an interference-limited mobile system, the geographic distribution of the SINR over the area of the cell is irregular. The normalized channel capacity for a typical hexagonal cell in a simulated system, with Rayleigh fast-fading and log-normal shadow fading having a standard deviation of $6 \mathrm{~dB}$ and a frequency of $1 \mathrm{~Hz}$, is shown in Fig. 1 when using the associated SINR - rather than SNR - in the approximate channel 
Table 4

Summary of System Features for the Reconfigurable Mobile Radio System (From [142])

PSA-BPSK: Pilot Symbol Assisted Binary Phase Shift Keying. PSAQAM: Pilot Symbol Assisted

Quadrature Amplitude Modulation. FEC: Forward Error Correction Coding. PSNR: Peak

Signal-to-Noise Ratio. TDMA/TDD: Time Division Multiple Access/Time Division Duplexing

\begin{tabular}{|c|c|c|c|c|}
\hline Features & \multicolumn{4}{|c|}{ Multimode System } \\
\hline Modem & PSA-BPSK & 4-PSAQAM & 16-PSAQAM & 64-PSAQAM \\
\hline Bit/Symbol & 1 & 2 & 4 & 6 \\
\hline Number of sub-channels & 1 & 1 & 2 & 3 \\
\hline C1 FEC & $\mathrm{BCH}(127,85,6)$ & $\mathrm{BCH}(255,171,11)$ & $\mathrm{BCH}(255,191,8)$ & $\mathrm{BCH}(255,199,7)$ \\
\hline $\mathrm{C} 2 \mathrm{FEC}$ & N/A & N/A & $\mathrm{BCH}(255,147,14)$ & $\mathrm{BCH}(255,163,12$ \\
\hline C3 FEC & N/A & N/A & N/A & $\mathrm{BCH}(255,131,18)$ \\
\hline Source bit rate (kbit/s) & 4.25 & 8.55 & 16.9 & 24.65 \\
\hline FEC-coded bit rate (kbit/s) & 7.3 & 14.6 & 29.2 & 43.8 \\
\hline Video PSNR (dB) & 32 & $\overline{35}$ & 37.5 & 39 \\
\hline Min. AWGN SINR (dB) & 4 & 10 & 15 & 20 \\
\hline Min. Rayleigh SINR $(\mathrm{dB})$ & 10 & 15 & 20 & 30 \\
\hline User Symbol Rate (kBd) & \multicolumn{4}{|c|}{7.3} \\
\hline No. of Users & \multicolumn{4}{|c|}{9} \\
\hline System Symbol Rate (kBd) & \multicolumn{4}{|c|}{131.4} \\
\hline System Bandwidth (kHz) & \multicolumn{4}{|c|}{200} \\
\hline Effective User Bandwidth $(\mathrm{kHz})$ & \multicolumn{4}{|c|}{11.1} \\
\hline TDMA frame length (ms) & \multicolumn{4}{|c|}{ 20; 8-slot fixed assignment } \\
\hline UL / DL Duplexing & \multicolumn{4}{|c|}{ TDD } \\
\hline Slots/Frame & \multicolumn{4}{|c|}{18} \\
\hline Vehicular Speed $(\mathrm{m} / \mathrm{s})$ & \multicolumn{4}{|c|}{13.4} \\
\hline Carrier Frequency $(\mathrm{GHz})$ & \multicolumn{4}{|c|}{1.8} \\
\hline Fast Fading Normalised Doppler Freq. & \multicolumn{4}{|c|}{$6.2696 \times 10^{-4}$} \\
\hline Log-Normal Shadowing & \multicolumn{4}{|c|}{ standard deviation: $6 \mathrm{~dB}$, freq.: $1 \mathrm{~Hz}$} \\
\hline Path loss Exponent & \multicolumn{4}{|c|}{3.5} \\
\hline BS station Separation $(\mathrm{km})$ & \multicolumn{4}{|c|}{1} \\
\hline
\end{tabular}

capacity formula proposed by Lee [76]. The horizontal coordinates $X$ and $Y$ indicate the distance from the BS of the 200-m-radius cell, while the location of the highest channel capacity peak in the center of the cell indicates the position of the BS. Six uplink interferers were used, all of which resided in different co-channel cells of the so-called first tier at random locations, since there were only six users communicating at the same frequency in the same time slot. This explains the nonsymmetric nature of the channel capacity profile in Fig. 1 over the area of a typical-approximately 400-m diameter-microcell, where the co-channel base stations (BS) are $1 \mathrm{~km}$ apart. All these propagation conditions are summarized in Table 4. Observe in Fig. 1 that the central portion of the cell exhibits typically higher maximum channel capacity than the fringe areas, which will be exploited by the programmable transceiver of Table 4, using 1-6 bits per symbol signaling. Note furthermore that these maximum channel capacity estimates are reduced proportionally by the Nyquist excess bandwidth, for example, in case of a modulation rolloff factor of unity by a factor of two.

Let us now concentrate our attention on the effects of co-channel interference inflicted by the adjacent cells using the same set of frequencies-an issue, which further justifies the employment of burst-by-burst adaptive transceivers [27]-[29].

\section{EFFECTS OF CO-CHANNEL INTERFERENCE}

The co-channel interference performance and the capacity of various cellular systems were investigated, e.g., by Lee and Steele in [77]. Our co-channel interference studies have mainly concentrated on the uplink of hexagonal cells with a reuse factor of seven, using an omnidirectional antenna at the center of each cell. This is a commonly investigated cellular cluster type, where each BS has six so-called first-tier co-channel interferers, as seen in Fig. 2.

The parameter that affects the co-channel interference most is the position of the interferers, which are constituted by the MSs in an uplink scenario. An interferer, which is moving away from its BS within a co-channel cell in the direction of the serving cell, will inflict an increasing interference. If power control is employed, this is likely to be aggravated by its increased transmitted power. However, in a typical scenario, where users are randomly distributed within a cell, power control is expected to reduce the interference load of the system. In order to investigate how the position of the interferers affects the co-channel interference, the following scenarios were investigated.

1) The least detrimental, i.e., "best" interferer position, where all the interferers are placed as far as possible from the interfered BS. This situation is illustrated for the stipulated propagation conditions of Table 4 in Fig. 3(a) in terms of the SIR versus distance from the BS.

2) Worst case interferer position, where all the interferers are positioned as close as possible to the interfered BS, which is characterized by Fig. 3(b).

3) Random interferer position, where interferers are allocated randomly within their cell, representing the most typical scenario. 

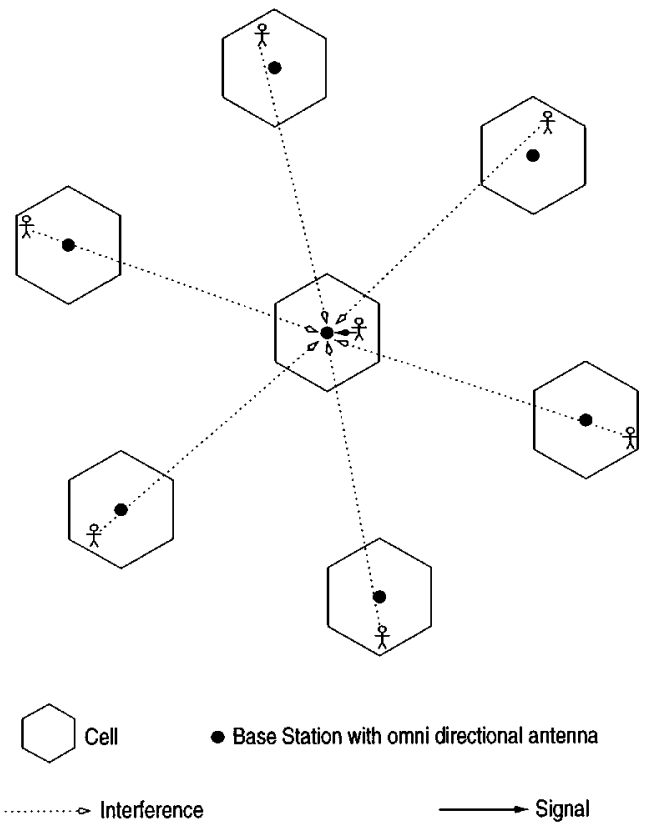

(a) Minimum Interference
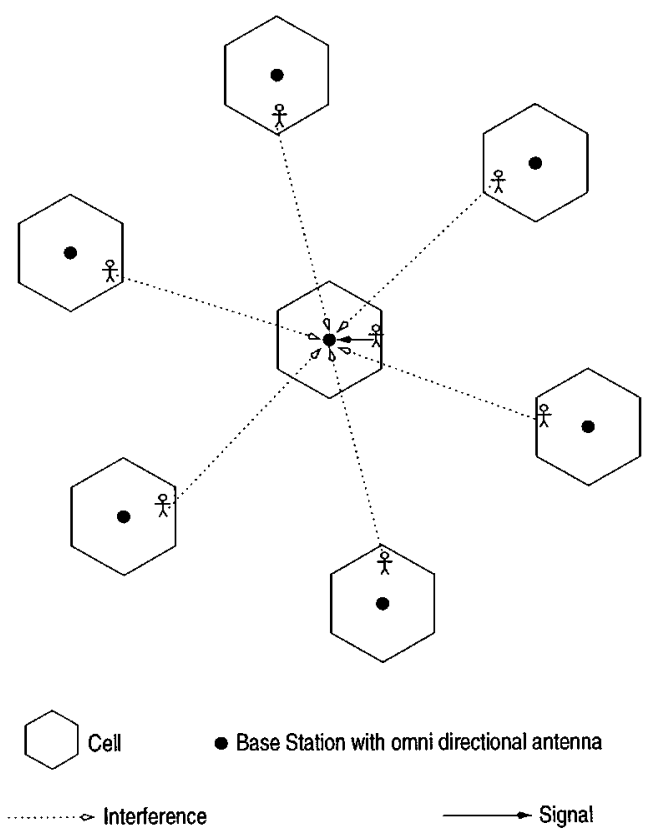

(b) Maximum Interference

Fig. 2. Signal and interference paths for uplink simulations, when interfering users are in the positions to cause minimum or maximum interference, for a seven-cell reuse cluster.

It was found that the SIR difference between the best and worst interferer positions varied, but at the edge of the cell was on the average about $6 \mathrm{~dB}$, which is also demonstrated by comparing Fig. 3(a) and (b). Again, the high-SIR central portion of the cell provides a higher maximum channel capacity, which will be exploited in terms of better video quality by our programmable transceiver of Table 4 . In case of voice-activity detection (VAD) [30] assisted speech transmissions, the above interference loads are reduced by about $4 \mathrm{~dB}$ due to the approximately halved on-air time.

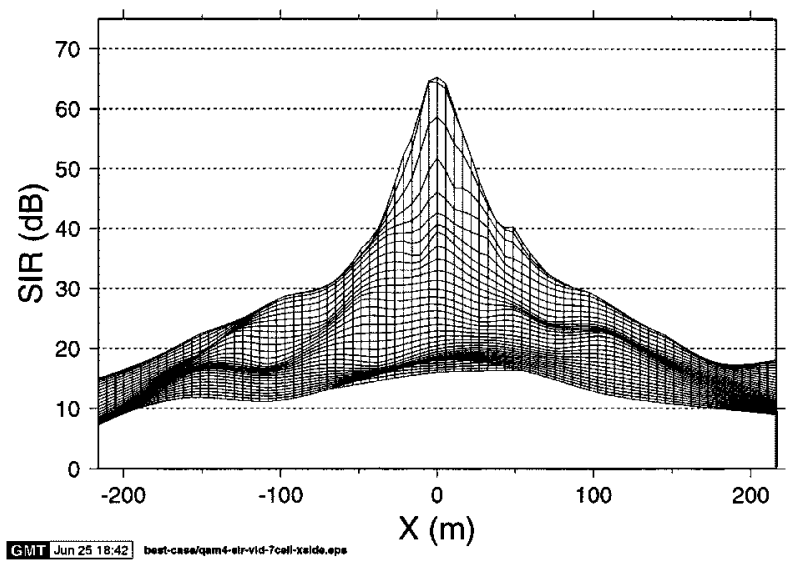

(a) Best Interferer Position

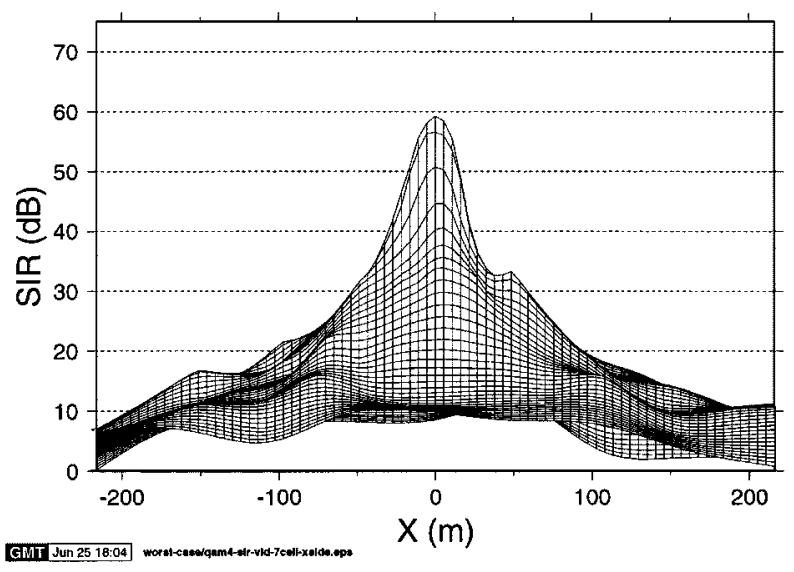

(b) Worst Interferer Position

Fig. 3. Simulated average SIR (dB) profile of a hexagonal cell without power control, employing a reuse factor of seven, path loss exponent of 3.5, slow-fading frequency of $1 \mathrm{~Hz}$, standard deviation of $6 \mathrm{~dB}, 4-\mathrm{QAM}$ modulation, and interference from six stationary video users in co-channel cells (from [142]).

Due to the fast fading and slow fading of both the signal and interference, the instantaneous SINR has a larger variance in interference-limited scenarios. This can be particularly adverse when the uplink signal is highly attenuated by a deep fade and, coincidently, the interference is boosted by the fading, resulting in a very low instantaneous SINR. The corresponding SINR profile evaluated across the cell area suggests that an intelligent transceiver can exploit the high channel quality of the central section of the cell, which supports the rationale of this contribution.

When using power control, the geographic distribution of the wireless channel capacity over the area of the cell varies less dramatically. However, there is a range of other propagation phenomena that gravely influence the instantaneous channel capacity on a more short-term basis. More specifically, in addition to the path-loss, slow-fading, and fast-fading related channel capacity fluctuations, the CCI and intersymbol interference (ISI) also exhibit short-term 
fluctuations. Most of these phenomena cannot be counteracted by the power control scheme alone. For example, power control is unable to combat signal dispersion inflicted by the path-length differences of the various propagation paths. Indeed, powering up in order to improve a particular user's power budget may disadvantage a number of other users, which in turn require power increments themselves, potentially leading to instability of the system. By contrast, burst-by-burst adaptive transceivers support a "nondestructive" transceiver adaptation to time-variant channel conditions.

The required relative frequency of transceiver reconfigurations still remains to be resolved, however. Ideally, each transmitted symbol ought to be transmitted in the "optimum" modem mode, allowing us to maintain the required transmission integrity expressed in terms of the BER or transmission burst error rate while maximizing the associated number of bits per symbol. This, however, would require a symbol-bysymbol based channel quality estimation, which is unrealistic in practical terms. By contrast, a call-by-call adaptation would be inadequate in transmission integrity terms, since the usual bursty channel error events of the conventional fixed-mode modems would be experienced. The actual channel quality fluctuations are a complex function of all the users' normalized Doppler frequency [30], which predetermines the rate of the associated CCI as well as ISI fluctuations. An informal insight into the rate of channel quality fluctuations associated with the channel impulse response to be introduced at a later stage in the context of Fig. 13 can be gained, also, by considering the modem mode switching example to be discussed in Fig. 14 of Section VIII-D.

Having informally characterized the propagation environment [30] and justified the utilization of burst-by-burst adaptive transceivers [27]-[29], let us now focus our attention on video transmission aspects, in particular, the design of an intelligent multimode videophone transceiver.

\section{SyStem DESIGN ASPECTS OF VIDEO TRANSCEIVERS}

\section{A. State-of-the-Art}

The state-of-the-art in this field has been portrayed, e.g., in the special issue [111] based on the invited contributions of [112]-[118]. Specifically, Girod and Färber [112] have been influencing the wireless video communications community and the research trends in the field for years, and in their contribution to this issue, they considered feedback-based video transmission arrangements. In an effort to reduce the sensitivity of the system to transmission errors, the proposed video decoders invoked a combination of error detection, resynchronization, and error concealment, which are applicable to most interframe coding-based video compression standards, such as the H.263 and MPEG4 schemes. In their contribution, Villasenor et al. [113] presented an overview of robust video compression techniques and systems in the context of the MPEG4 standard, for example. Van Dyck and Miller [114] contributed in the field of joint video source and channel coding, an area that has attracted much attention in the recent literature, especially when amalgamating these standard video-codec-based techniques also with the modem employed.

Gharavi and Alamouti [115] advocated employing bit-error sensitivity motivated video bit stream partitioning, in order to more strongly protect the more error-sensitive synchronization and bit stream syntax-related header information of standard video codecs, such as H.263 and MPEG2, for example. More explicitly, their proposed twin-class error-sensitivity-based partitioning arrangement adaptively controlled the separation of the variable-length coded discrete cosine transform (DCT) coefficients and the above-mentioned synchronization- and header-related bits, in an effort to maintain a near-constant bit rate. The authors invoked the IMT-2000 CDMA system in order to characterize the performance of their proposed arrangement. Hagenauer and his colleagues have pioneered intelligent channel coding techniques [116] over the years, contributing to a vast range of advances in the field. In line with Van Dyck and Miller, in their contribution to this special issue, Hagenauer and Stockhammer [116] concentrate mainly on the employment of joint source and channel coding techniques, emphasizing that the system architecture has to be able to adapt to the hostile channel conditions. Focusing more on transmission aspects, Rohling et al., who have been spearheading many of the recent multicarrier transmission [28], [148] based research initiatives, review a range of aspects associated with high-rate multimedia, multicarrier radio transmissions to mobile receivers. Raychaudhuri, who has been instrumental in the conception and development of wireless asynchronous transfer mode (WATM) systems constituting a tetherless extension of the existing ATM backbone, offered a portrayal of the recent developments in this field. These high-rate WATM systems have the potential of delivering high-quality video to laptop computers, for example. Following a similar line of thought, ATM-based multicode CDMA was favored for the transport of MPEG-2 compressed video services over bandlimited mobile channels by Chang and Lin [118]. Similarly to the contribution by Gharavi [115], twin-class channel coding was proposed in order to protect the header and payload of ATM cells.

\section{B. Multimode Video Transceivers}

Following the above brief overview of some of the recent advances in the field, in this section, we provide a system design example in the context of an H.263-based intelligent multimode wireless TDD video transceiver. As argued above, TDD - rather than FDD - is predicted to be the more promising duplexing mode of the forthcoming $3 \mathrm{G}$ mobile radio standards due to its ability to support asymmetric traffic, which is deemed to be the most important scenario for the emerging World Wide Web (WWW)-based applications, such as Web browsing, etc. More explicitly, in file downloading, for example, one may require a single click on the uplink while dumping large files to the mobile terminal on the downlink. This allows the system to support potentially nearly twice as many users in a given bandwidth as the FDD mode, where the idle uplink cannot be efficiently exploited. Apart from the above advantages of TDD, it is 
also attractive for supporting burst-by-burst adaptive duplex operation, since, for example, the downlink channel quality estimate generated by the mobile receiver can be signaled to the transmitter of the BS in the immediately adjacent time slot of the TDD structure. This minimizes the delay between evaluating the perceived channel quality and adjusting the modem mode of the transmitter accordingly. These latency aspects were discussed by Torrance et al. [96], while the associated interference aspects were the topic of [97]. Hence, in this paper we concentrate on employing TDD.

As mentioned above, we target our system design example on a GSM-like H.263-based wireless video transceiver, noting that the design principles related to using multimode transceivers relying on adaptive video buffering and packetization are generically applicable-including, e.g., MPEG4-irrespective of the specific target bit rate or video resolution required. Embedding the system design guidelines in this specific, well-understood GSM-like context is likely to prove more tangible for the potential readership than simply phrasing them in generic terms.

An intelligent multimode transceiver is expected to exploit the potentially higher channel capacity of the central section of the propagation cell of Fig. 1, supporting higher quality video communications under higher SINR conditions while employing more error-resilient, but lower video-quality, modes under reduced SINR conditions near the fringes of the cell. This approach was adopted by Williams et al. [78], [79] on a static basis, although it can be invoked also on a more dynamically adapted burst-by-burst basis, as it is highlighted below.

Based on the observation that the instantaneous channel quality expressed in terms of SINR is time-variant in wireless systems, such burst-by-burst adaptive multimode, multilevel modems were invented by Steele and Webb at the University of Southampton in [80], [81], and [28] for slowly fading wireless pedestrian channels, where the channel fluctuations were slow with respect to the transmission burst length. Their work spawned further research in recent years [87]-[95] worldwide. Specifically, Goldsmith and her team [82], [85] at Stanford University, Pearce et al. [90] at York University, Kamio et al. at the University of Osaka and the Ministry of Post in Japan [86]-[89] contributed further to this field.

The proposed schemes provide a means of realizing some of the time-variant channel capacity potential of the fading wireless channel [2], [84], invoking a more robust transmission scheme (TS) on a burst-by-burst basis, when the channel is of low quality. In [91], Torrance et al. derived the analytical upperbound performance of such an adaptive quadrature amplitude modulation (AQAM) scheme over slow Rayleigh-fading channels, while in [94], an unequal protection phasor constellation for signaling the transmission scheme of the modem was proposed. The problem of appropriate power assignment in AQAM modems was discussed, e.g., by Morimoto et al. [88] and by Chua and Goldsmith [82]. We note that AQAM can outperform any of its constituent fixed modem modes in BER terms, since it performs always at least as well as the constituent modem modes but on average transmits a higher number of bits per symbols (BPS) due to assigning as high a number of bits per symbol under favorable instantaneous channel conditions, as possible, while satisfying the required BER constraints. Furthermore, fixed modems cannot refrain from transition, when the channel quality is low, and, hence, bursts of errors are inflicted, which potentially overloads the error correction capability of the associated forward error correction (FEC) codecs. By contrast, a substantial advantage of AQAM is that it can reduce the number of bits per symbol until the target BER requirement is met. Due to this, the FEC codec benefits from less bursty error statistics, which increases the achievable coding gain.

In [93], a combined BER and BPS based optimization cost function was defined and minimized by Torrance $e t$ al.in order to find the required modulation switching levels for maintaining average target BERs of $1 \times 10^{-2}$ and $1 \times 10^{-4}$, irrespective of the instantaneous channel SNR. The former scheme was referred to as the speech scheme and the latter one as the adaptive data scheme. Since disabling transmission during deep fades results in a controllable latency, in [95] and [98] the latency performance of these schemes was quantified and frequency hopping as well as statistical multiplexing were proposed to mitigate the latency inflicted and to reduce the buffer requirements. The expected performance benefits of such burst-by-burst adaptive modems over wide-band channels were evaluated by Wong et al. [99] upon defining a novel channel quality metric based on the mse of the channel equalizer employed.

As seen in Table 4, our design example employed here uses embedded binary Bose-Chaudhuri-Hocquenghem $(\mathrm{BCH})$ coding [141] combined with a reconfigurable pilot symbol assisted (PSA) quadrature amplitude modulation (QAM) modem [28]. Here, we refrain from detailing the $\mathrm{BCH}$ coding and QAM issues; the interested reader is referred to [141] and [28] for an in-depth discourse. We note, however, that the performance of the system can be further improved by employing turbo channel coding, which was invented by Berrou et al. [58], [59].

Returning to the proposed multimode video transceiver, which is capable of adapting to time-variant channel condition, it can operate under network control in one of four modes, corresponding to 1-, 2-, 4-, and 6-bit/symbol modulation schemes. This allows the system to span a wide range of operating conditions in terms of video quality, bit rate, robustness against channel errors, and implementational complexity while exploiting the higher channel capacity of the central region of Fig. 1. For example, the transceiver operates using highly bandwidth-efficient 64-level PSAQAM (64-PSAQAM) in a benign indoor cordless environment, where high SNRs and SIRs prevail. The number of modulation levels is dropped from 64 to 16, when the portable station (PS) is handed over to an outdoor street microcell, and can be further reduced to four or two in less friendly propagation scenarios. Again, the system parameters are summarized in Table 4.

The video packetization algorithm of [142], which will be highlighted during our forthcoming discourse, was in- 
voked in order to ensure that the H.263 codec generated a near-constant bit rate-upon invoking different quantizers from the legitimate set of quantizers in the H.263 scheme [142] - spanning a bit rate range of a factor of 1-6, corresponding to the 1-6 bit/symbol QAM modem modes. Hence, the FEC coded signaling rate became $7.3 \mathrm{kBaud}(\mathrm{kBd})$ in all modes. We use here Bd-rate, rather than bit rate, since constant bandwidth multimode transceivers maintain a constant Bd-rate but their bit rate varies, depending on the number of bits per symbol transmitted. When opting for a modulation excess bandwidth of $50 \%$ and a system bandwidth of 200 $\mathrm{kHz}$, as in the GSM system, the maximum signaling rate becomes $133.33 \mathrm{kBd}$. At this signaling rate, INT(133.33/7.3) $=18$ time slots can be created, where INT indicates the integer quotient of the division. Assuming an identical speech signaling rate of $7.3 \mathrm{kBd}$, nine videophone users can be supported by the proposed scheme in the $200-\mathrm{kHz}$ bandwidth of the GSM system. A range of further system aspects can be inferred from Table 4, but due to space limitations here, we refrain from detailing them; they are left for the interested reader to explore in more depth [27], [142].

Having reviewed the salient features of an intelligent multimode transceiver, we emphasize again that our objective was to highlight the system design philosophy, which is applicable not only to the specific system design example used here, but also to arbitrary video bit rates, video resolutions, and a range of other parameters. Let us now briefly consider the aspects of our advocated packetization algorithm, which was detailed in [142]. The algorithm proposed exhibits high flexibility, supporting both automatic repeat request (ARQ), a technique documented by Lin et al. in [143] and packet dropping, although in Sections VI-VIII we will present results for the lower delay, lower complexity scenario of packet dropping rather than retransmitting corrupted video packets. Due to the bit error sensitivity of the H.263 codec, corrupted packets cannot be used by either the local or the remote decoder since that would result in unacceptable video degradation due to error propagation. Hence, these corrupted packets must be either retransmitted or dropped. For ARQ-assisted operation, the corrupted packets may have to be reencoded at a lower bit rate for more robust retransmission [27], while for video packet dropping, both the local and remote reconstruction buffers are updated by a blank packet. A key aspect of our proposed regime is, therefore, the provision of a strongly error protected binary transmission packet acknowledgment flag [142], which instructs the remote decoder not to update the local and remote video reconstruction buffers in the event of a corrupted packet. This flag can be, for example, conveniently repetition-coded, in order to invoke majority logic decision (MLD) at the decoder. Explicitly, the binary flag is repeated an odd number of times, and at the receiver the MLD scheme counts the number of binary ones and zeros and decides upon the logical value, constituting the majority of the received bits. These repeated packet acknowledgment flags are then superimposed on the forthcoming reverse-direction packet in our advocated TDD regime of Table 4. These system aspects will become more explicit in the context of Fig. 4 during our forthcoming elaborations.
We opted for using an MLD code of $\operatorname{MLD}(27,1,13)$, implying that the 1-bit flag was repeated 27 times and, hence, up to 13 transmission errors can be corrected. This corresponds to a channel BER of about $50 \%$, and in our investigations acknowledgment errors were only encountered at video frame error rates of around $100 \%$. The corresponding framing structure was shown explicitly by Keller et al. in [146] and [147] in the context of a TDD/OFDM WATM system, where the superimposed acknowledgment flag was portrayed along with the associated delay ramifications. For a detailed treatise on WATM developments, the reader is referred, e.g., to the work by Raychaudhuri and Wilson [157], [196], [158], and Toh [159]. We emphasize again that the design principles transpiring from this paper in the context of low-rate video codecs are equally applicable to the high-rate WATM arrangements of [146] and [147]. A range of multiple access schemes amenable to variable-rate multimedia statistical multiplexing were proposed, e.g., by Brecht et al. in [160]-[162]. In our proposed system, the associated acknowledgment feedback flag is transmitted within a TDD frame duration of $20 \mathrm{~ms}$, which is a small fraction of the 100 -ms video-frame refreshment interval encountered in the case of a video frame rate of 10 frames/s.

An example of this transmission feedback signaling and timing is shown in Fig. 4 in the context of the system used in our design example. Upon receiving a transmission packet, the transceiver has two tasks to perform before the next transmission. First, the video datastream has to be demodulated and FEC decoded in order to check whether there were errors in the video stream, since this event has to be signaled in the next transmission burst to the transmitter. The second task is to produce the next video packet for the forthcoming transmission burst, as indicated in Fig. 4. The next packet of video data cannot be encoded until the feedback signaling for the previous transmission is received. This is because the motion vectors used in the next video data packet may depend on blocks transmitted in the previous packet. Once the feedback acknowledgment flag of the previous video packet's transmission is received, which was superimposed on the reverse channel's video packet, the effect of the loss or success of the previous video packet can be taken into account in the local decoder of the H.263 codec. Explicitly, if the previous packet was lost, the effective changes made to the local video decoder for this packet are discarded. By contrast, if the previous packet was received without errors, then the local decoder update pertinent to the previous packet is made permanent. If ARQ is used, then the transmission feedback signaling may have requested a reencoding of the packet at the same or a lower bit rate. In this case, the reconstruction buffer changes related to the previous packet are discarded and the codec parameters are modified in order to reduce the video bit rate temporarily. Once the feedback signaling was invoked in order to synchronize the reconstruction frame buffers of the local and remote H.263 codecs, the H.263 codec starts to encode the next video packet, as indicated in Fig. 4.

The time between receiving a packet and transmitting a new packet is approximately half the frame length $T$. Since 


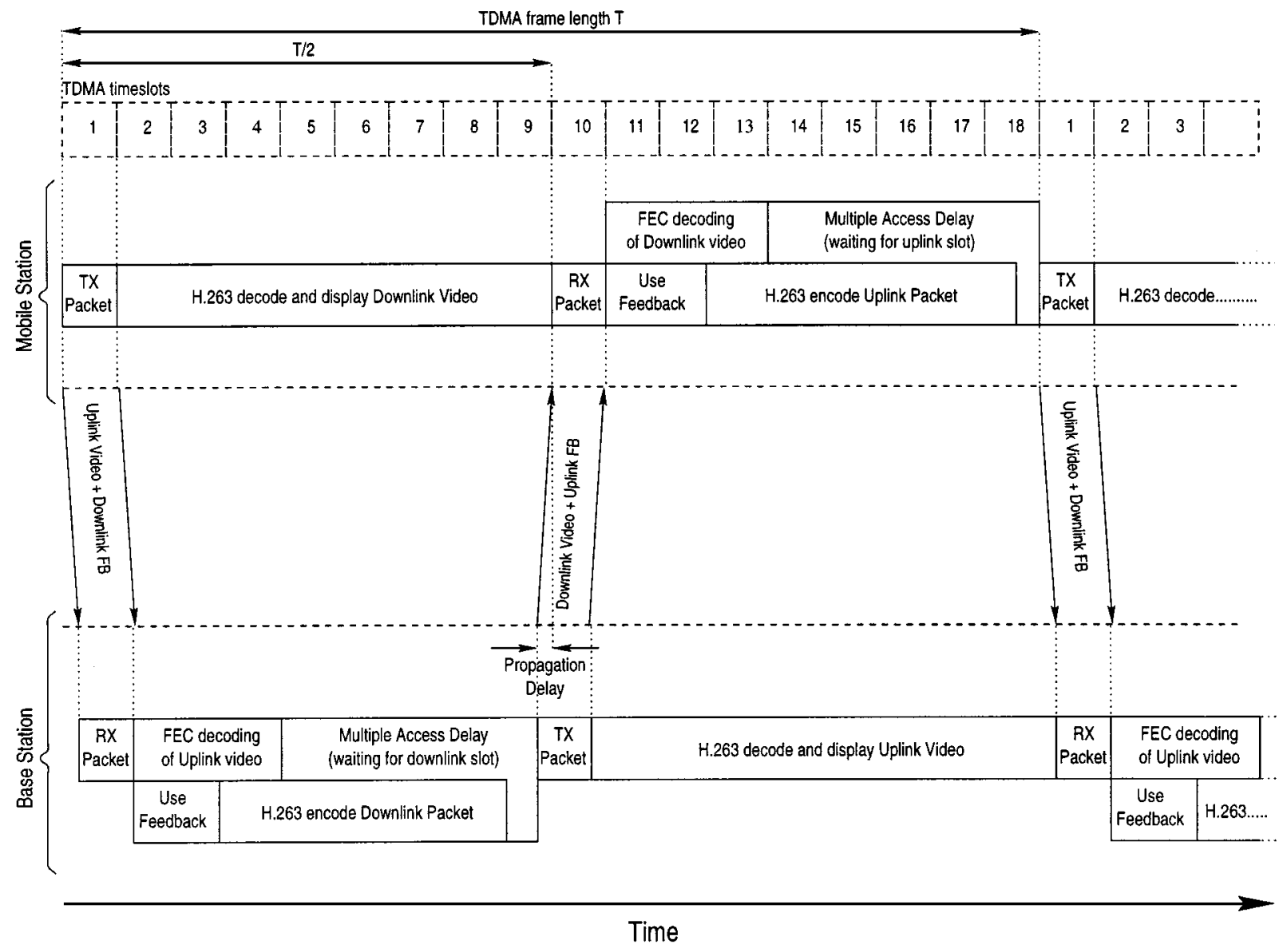

Fig. 4. Transmission feedback timing, showing the feedback signaling superimposed on the reverse channel video datastream. The tasks that have to be performed in each time interval are shown for both the mobile station and the base station.

we are considering microcells, the propagation delays are very low; e.g., for a distance of $1 \mathrm{~km}$, the delay is $3.3 \mu \mathrm{s}$. Within the time period $T / 2$ between receiving and transmitting packets, the transceiver has to calculate the feedback signaling for the received packet and to generate a new video packet for transmission. These two tasks can be done in parallel or in series, but they must be completed within the $T / 2$ time period. After a packet is transmitted, there is another $T / 2$ time period, before the next received packet arrives. This time period, as shown in Fig. 4, is used to decode the H.263 video data received in the previous packet and to update the video display.

We note that invoking ARQ in order to enhance the robustness of the system is a more realistic option for interactive video communication in short framelength WATM schemes [146], [147] than in the GSM-like video transceiver considered here, due to the associated lower latency. Again, this scenario was exemplified in [146] and [147]. In order to improve the flexibility of TDD schemes, Brecht et al. suggested the employment of statistical packet assignment multiple access (SPAMA) [162] for supporting variable- or fixed- but programmable-rate multimedia traffic. The statistical nature of the proposed centralized slot assignment scheme facilitated an accurate matching of bit-rate requirements for different rate video services with a minimal amount of sig- naling, while maintaining a throughput of up to $93 \%$ at the cost of a low delay, where throughput was defined as the proportion of time during which the transmission medium was actively engaged in communications. A further alternative to support similar multirate multimedia users was also proposed by Brecht et al., which was termed multiframe PRMA (MF-PRMA) [161], [160].

Before we embark on choosing a video codec and characterizing its performance, we note that for an intelligent multimode transceiver, a source codec is required, which is capable of conveniently exploiting the variable-rate, timevariant capacity of the wireless channel by generating always the required number of bits that can be conveyed in the current modem mode by the transmission burst. During use of a given modem mode associated with a constant bit rate, the packetization algorithm employed in our design example maintains a constant bit rate in conjunction with the bit-rate control of the codec. These aspects are considered along with further video compression issues in the next section.

\section{LOW-RATE VIDEO CODING ASPECTS}

\section{A. Advances in Low-Rate Video Compression}

The pivotal issue in designing videophone schemes is finding the most attractive video codec in terms of video 
quality, bit rate, implementational complexity, robustness against channel errors, coding delay, bit-rate fluctuation and the associated buffer length requirement, etc. Most of these video compression issues were treated in a range of classic books by Jayant and Noll [101], Clarke [102], Netravali et al. [103], [104], Keith [105], Woods [126], Jain [106], Rao and Yip [107], Tekalp [108], and Gersho and Gray [172]. A variety of video compression schemes have been suggested in the special issues edited by Gharavi et al. [109]-[111], Tzou et al. [119], Hubing [120], Girod et al. [121], and Zhang et al. [169] for a range of bit rates, video resolutions, and applications.

In terms of standard video compression schemes, the MPEG1, MPEG2 [49], [50], and $p \times 64 \mathrm{~kb} / \mathrm{s}$ ITU H.261, H.263 video codecs [27], [53] have been contrived for high-rate low-BER fixed channels. Although the MPEG4 working group's activities also target mobile videophony [169] and work is under way toward defining a so-called mobile extension to the H.263 codec, to date, there are no approved video coding standards for mobile videophony over existing or future standard radio systems. Khansari et al. [130] as well as Pelz [163] reported promising results on adopting the H.261 codec for wireless applications by invoking powerful signal-processing and error-control techniques in order to mitigate the error sensitivity problems due to stretching the application domain of these vulnerable run-length compressed schemes to hostile wireless environments.

In the new MPEG4 codec [169], a range of optional tool-box oriented techniques can be employed in order to provide scope for future innovation, e.g., by using so-called object-oriented coding techniques. In this context, a video object is an arbitrary entity, such as, for example, the image of a computer, a plant, or the limbs of a person in the video frame, and these objects jointly form a picture scene. In addition to such video objects, it is also possible to define analogous audio objects, such as a person's voice, music, a dog barking in the background, etc. In this context, a unique feature of MPEG4 in comparison to existing audio-visual standards is the definition of such so-called audiovisual objects, which are associated with spatial and temporal coordinates.

In low-rate object-based analysis by synthesis coding studied by Chowdhury et al. [122], attempts are made to characterize each moving object by their shape, texture, and motion characteristics, which can be combined with more conventional methods in order to further reduce the bit rate. In the knowledge-based or object-based coding approach advocated by Bozdagi et al. [123], a priori knowledge with regards to the shape of the object to be encoded can be exploited in order to represent, for example, a person's head by means of its so-called wire-frame model. The motion of an object, such as the head, can be classified as global and local, corresponding to head movements and various facial expressions, respectively. These translations can be represented by fractal transformations [129] of previously coded so-called range blocks. Analysis by synthesis coding, which was investigated, e.g., by Choi et al. [124], typically attempts to synthesize the image to be encoded a number of times in order to arrive at the subjectively most attractive quality versus bit-rate tradeoff. This inevitably increases the complexity and raises the question of finding an appropriate objective quality measure, which exhibits a high correlation with the subjective quality.

Färber et al. at the University of Erlangen also contributed substantially to the state-of-the-art by contriving various error-resilient H.263-based schemes [131]-[135] as well as innovating in motion compensation [136], [137]. Other valuable contributions in the area of motion estimation and compensation techniques are due to, e.g., Magarey and Kingsbury at Cambridge University [153], Czerepinski and Bull at the University of Bristol [154], and Nieweglowski et al. [155]. The error-resilient entropy coding (EREC) algorithm of Redmill and Kingsbury constitutes an important milestone toward improving the bit-error sensitivity of variable-length coded video [156], facilitating the employment of run-length coded standard MPEG-based [50] or H.263-based video communications. Eryurtlu et al. [138], [139] from the University of Surrey in the U.K. proposed various improvements to the H.263 scheme. Further important contributions in the field were due to Chen et al. [164], Illgner and Lappe [165], Zhang [166], Ibaraki et al. [167], and Watanabe et al. [168]. Vector quantization arrangements were favored by Ramamurthy and Gersho [173] as well as by Torres and Huguet [174].

Continuing our overview of nonstandard low-rate coding schemes [27]-[35], DCT [106]-based codecs were proposed in [27] and [32], while various subband coded solutions were advocated by Stedman et al. [125], Woods [126], Gharavi [127], and Ngan [128]. Both of these codec classes operate in the spatial frequency domain by decomposing the image in various frequency-domain components, and their components are assigned the appropriate number of bits according to the target bit rate aimed for. Motivated by the flexibility of subband codecs in terms of controlling the allocation of the quantization distortion to the various spatial frequency bands at the early stages of videophony research during the early 1990s, Stedman et al. [125] designed a $55-\mathrm{kb} / \mathrm{s}$ subband $\operatorname{codec}$, which was embedded in a $22-\mathrm{kBd}$ single-user 16-QAM transceiver. Following these advances, work evolved further by recognizing some of the limitations of the subband codec in terms of bit-rate reduction and motion activity tracking within the video frame. The $55-\mathrm{kb} / \mathrm{s}$ average rate was achieved with the aid of run-length coding, which is known to exhibit extreme sensitivity against channel errors, since a single error is sufficient to corrupt an entire encoded run [106] or even a video frame. In order to smooth the bit-rate fluctuation, adaptive-feedback buffering was used, which increased the codec's delay. Since, over wireless channels, the typical BER is high, below a threshold channel SNR, the system's performance broke down and became unacceptable. With regards to motion activity tracking within the video frame, we understood that in head-and-shoulders videophony there is typically a high proportion of low-activity background segments, which are amenable to higher compression ratios. 
Table 5

Picture Formats Supported by the H.261 and H.263 Codecs

\begin{tabular}{|c|c|c|c|c|c|c|c|c|}
\hline \multirow[t]{3}{*}{$\begin{array}{l}\text { Picture } \\
\text { Format }\end{array}$} & \multirow[t]{3}{*}{$\begin{array}{c}\text { Luminance } \\
\text { columns }\end{array}$} & \multirow[t]{3}{*}{$\begin{array}{c}\text { Luminance } \\
\text { lines }\end{array}$} & \multirow[t]{3}{*}{$\begin{array}{c}\text { H.261 } \\
\text { support }\end{array}$} & \multirow[t]{3}{*}{$\begin{array}{l}\text { H.263 } \\
\text { support }\end{array}$} & \multicolumn{4}{|c|}{$\begin{array}{c}\text { Uncompressed } \\
\text { bit rate (Mbit/s) }\end{array}$} \\
\hline & & & & & \multicolumn{2}{|c|}{$10 \mathrm{frame} / \mathrm{s}$} & \multicolumn{2}{|c|}{30 frame $/ \mathrm{s}$} \\
\hline & & & & & Grey & Colour & Grey & Colour \\
\hline SQCIF & 128 & 96 & No & Yes & 1.0 & 1.5 & 3.0 & 4.4 \\
\hline QCIF & 176 & 144 & Yes & Yes & 2.0 & 3.0 & 6.1 & 9.1 \\
\hline CIF & 352 & 288 & Optional & Optional & 8.1 & 12.2 & 24.3 & 36.5 \\
\hline $4 \mathrm{CIF}$ & 704 & 576 & No & Optional & 32.4 & 48.7 & 97.3 & 146.0 \\
\hline $16 \mathrm{CIF}$ & 1408 & 1152 & No & Optional & 129.8 & 194.6 & 389.3 & 583.9 \\
\hline
\end{tabular}

Based on these findings, the above-mentioned activity tracking approach was exploited by defining "motion active" and "DCT-active" blocks in [32], which led to a very high bandwidth efficiency while maintaining good quarter common intermediate format (QCIF) video quality-associated with a $144 \times 176$ pixel resolution-at 8.52 and 11.36 $\mathrm{kb} / \mathrm{s}$ and $10 \mathrm{frames} / \mathrm{s}$. Although the bit rate was constant, the $8.52-\mathrm{kb} / \mathrm{s}$ codec achieved this extremely low rate at the price of higher vulnerability to channel errors due to variable-length coding, while the $11.36-\mathrm{kb} / \mathrm{s}$ codec had a higher robustness against channel errors but slightly reduced compression efficiency. A common feature of both schemes was a slight video blurring due to the bandwidth-limiting nature of the DCT masking. In a further attempt to address this problem and to document the performance of potential benchmarkers under identical circumstances, Streit $e t$ al.also studied an 11.36-kb/s quadtree (QT) codec [33] and an identical-rate vector-quantized (VQ) codec [31].

The above fixed-rate DCT-, QT-, and VQ-based schemes were compared to the variable-rate ITU H.261 and MPEG2 codecs by Streit $e t$ al. in [34] in the context of their applicability to wireless videophony over existing $2 \mathrm{G}$ cellular and cordless mobile systems. The conclusion was that the best performance in terms of ensuring a fixed bit rate at the cost of no buffering latency, a good subjective video quality, low complexity, and high error resilience was offered by the VQ codec. By contrast, in this contribution, we report on adaptive postcoding-type video packetization techniques and appropriate transmission regimes, in order to render the H.263 ITU codec suitable for error-resilient, low-latency interactive wireless videophony over existing and future mobile radio systems.

\section{B. Video Sequences}

Again, the H.263 codec was detailed in [27], [53], and [175], while a number of transmission schemes designed for accommodating its rather error-sensitive bit stream were proposed, e.g., by the prestigious video communications groups led by Girod at Stanford University, Kondoz at Surrey University, Bull at Bristol University, and others, e.g., in [130]-[147]. As an illustrative example, in Table 5, we summarized the various video resolutions supported by the H.261 and H.263 ITU codecs in order to demonstrate their flexibility [27]. Their uncompressed bit rates at frame scanning rates of both 10 and 30 frames/s and for both gray
Table 6

Video Sequences Used for H.263 Simulations

\begin{tabular}{l|l|c|c}
\hline Video Clip & Size & Frame/s & Colour \\
\hline Miss America & QCIF & 10,30 & Grey \\
Miss America & SQCIF, QCIF, CIF & 10,30 & Colour \\
Carphone & QCIF & 10,30 & Colour \\
Suzie & SQCIF, QCIF, 4CIF & 10,30 & Colour \\
Football & 4CIF & 10,30 & Colour \\
Mall & 16CIF & 10,30 & Colour \\
\hline
\end{tabular}

and color video are also listed. The mature H.261 standard defined two different picture resolutions, namely, the $144 \times$ 176 pixel QCIF and the $288 \times 352$ pixel CIF, while the H.263 codec has the ability to support five different resolutions. All H.263 decoders must be able to operate in sub-QCIF (SQCIF) and QCIF modes and optionally support $\mathrm{CIF}, 4 \times \mathrm{CIF}$, and $16 \times \mathrm{CIF}$ formats. We note, however, that for cellular and cordless systems, only the $96 \times 128$ pixel SQCIF and the QCIF resolutions are realistic in terms of their minimum required bit rates. Hence, in this contribution, we will concentrate mainly on using QCIF sequences.

\section{H.263 Codec Performance}

In this section, we will report our findings with regards to the tradeoffs between image quality and bit rate for different image sizes, frame rates, and video sequences when using the H.263 codec, in order to demonstrate its ability to support the operation of intelligent multimode transceivers. All simulations were conducted using well-known video sequences at both 10 and 30 frames/s. The video sequences used are summarized in Table 6. The image resolutions employed were described in Table 5. Due to the bit-rate limitations imposed by cellular and cordless systems, here we will mainly concentrate our discussions on the smaller SQCIF and CIF formats and refer the interested reader to [26], [146], and [147] for further results concerned with high-resolution, high-rate WATM-type systems using an OFDM modem and binary $\mathrm{BCH}$ as well as turbo channel codecs.

1) Grayscale Versus Color Comparison: In this section, we embarked on investigating the extra bit rate required to support color video coding, as opposed to grayscale images. The grayscale sequences were generated by extracting the luminance information from the color video sequence. The results of our gray versus color investigations are portrayed in Fig. 5. The corresponding graphs characterize the image 


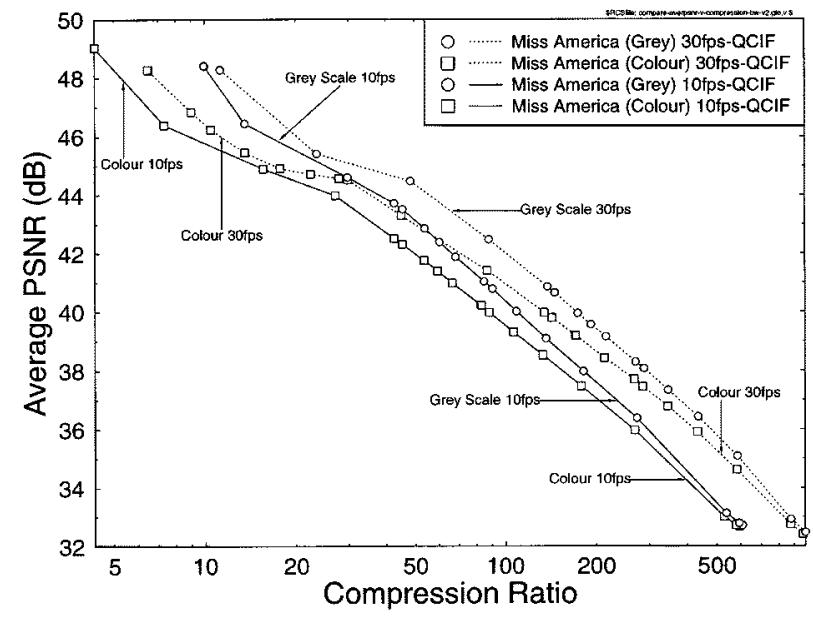

Fig. 5. Image quality (PSNR) versus compression ratio performance of the H.263 codec for the QCIF "Miss America" sequence in color and grayscale at 10 and 30 frames/s.

quality expressed in terms of peak signal-to-noise ratio (PSNR) ${ }^{4}$ versus bit rate and compression ratio, respectively. These graphs suggest that the grayscale and color PSNR performances are rather similar, but their difference becomes slightly more dominant with increasing bit rate. These tendencies imply that assuming a constant bit rate, only a small fraction of the overall bit rate has to be allocated to convey the color information, and, therefore, the PSNR penalty due to reducing the bit rate and resolution of the luminance component, required to accommodate the color information is marginal.

2) Comparison of QCIF Resolution Color Video: The investigations in this section were conducted in order to characterize the performance of the H.263 codec for a range of different video sequences. All simulations were carried out using color video sequences scanned at 10 and 30 frames/s. The video sequences used were "Miss America," "Suzie," and "Carphone." Some example QCIF video sequences can also be viewed on the Web. ${ }^{5}$

The results of these simulation-based experiments are plotted in Fig. 6. As expected on the basis of its low motion activity, the graphs suggest that the "Miss America" sequence is the most amenable to compression, followed by "Suzie," and then the "Carphone" sequence. The achievable compression ratios span an approximately one order of magnitude range when comparing the lowest and highest activity sequences. Due to lack of space here, we have to curtail our elaborations on the rather instructive further aspects of the H.263 scheme using, for example, different resolution sequences. A range of further bit-error sensitivity and transmission aspects at various resolutions are detailed in [27], while for further results concerned with high-resolution, high-rate WATM-type systems using an OFDM

\footnotetext{
${ }^{4}$ The PSNR is an often used video quality measure, which is similar to the conventional SNR, with the exception that the video reproduction error energy due to the coding impairments is normalized not to the actual signal energy but to the energy of a hypothetical video frame, where all pixels have the luminance value of 255 .
}

5http://www-mobile.ecs.soton.ac.uk/peter/h263/h263.html\#Examples.

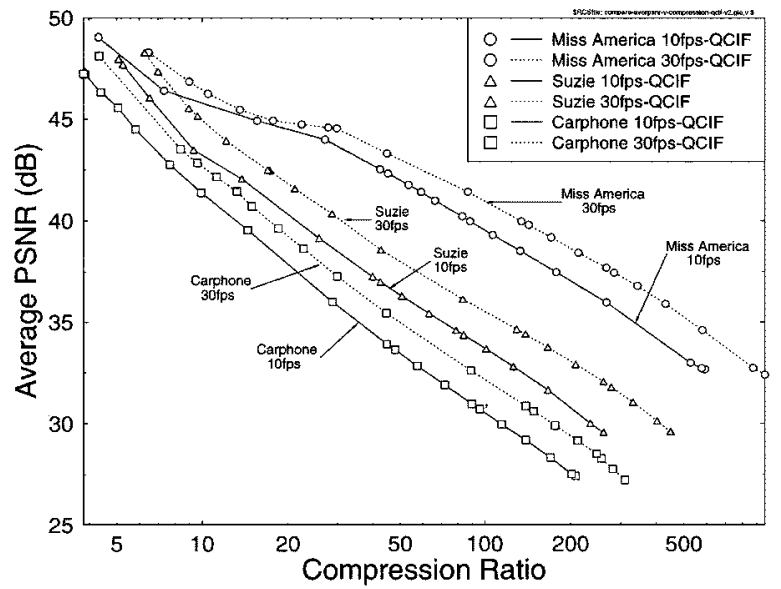

Fig. 6. Image quality (PSNR) versus compression ratio performance of the H.263 codec for QCIF resolution at 10 and 30 video frames/s.

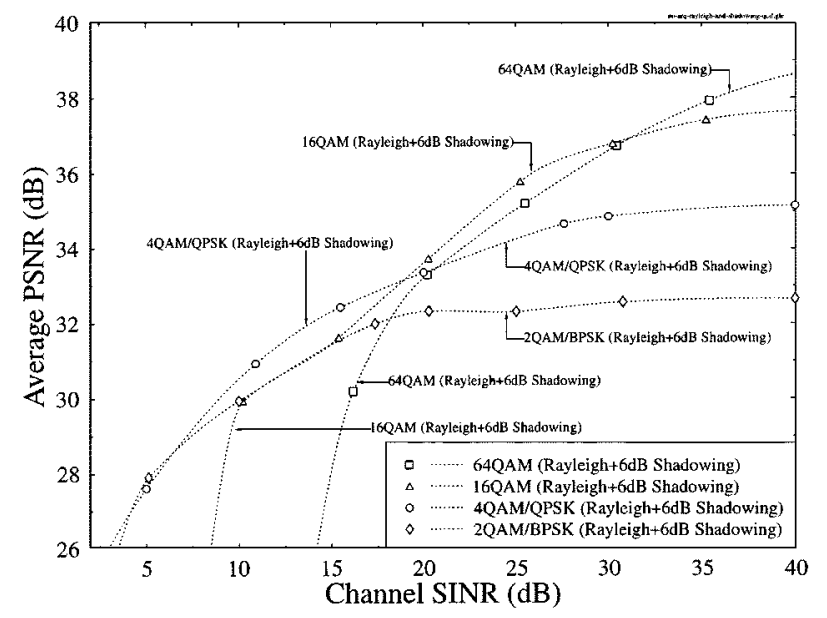

Fig. 7. Decoded video PSNR versus channel SNR performance for transmissions over Rayleigh-fading channels with 6-DB shadow fading using BPSK, QPSK, 16 QAM, and 64 QAM (from [142]).

modem and binary $\mathrm{BCH}$ as well as turbo channel codecs, the interested reader is referred to [27], [146], and [147].

\section{Video Performance Without Power Control}

In this section, we characterize the expected video system performance of the prototype scheme employed, when using no power control, a scenario often encountered in the cordless telephone schemes of Table 1, i.e., in DECT, CT2, etc.

The PSNR versus average SINR performance of the various transceiver modes is portrayed in Fig. 7 in conjunction with shadow fading exhibiting a standard deviation of $6 \mathrm{~dB}$. In systems, where the erroneously channel decoded transmission bursts are discarded in an effort to prevent channel error propagation to future video frames through the reconstructed video frame buffer due to the motion-compensation assisted interframe coding, the transmission burst error rate (BurstER) was found to be a reliable parameter in controlling the modem modes [27]. It was found advantageous to invoke a more robust modem mode typically around BurstER = $5 \%$, which was reached before actually the reduced PSNR of 
a less robust mode due to channel errors dropped below that of the next more robust mode exhibiting an inherently lower, but unimpaired, PSNR. In other words, this implied that a lower PSNR — which was unimpaired however by channel effects-was typically preferable to an originally higher, but channel-impaired, scenario. The associated subjective effects are characterized by the corresponding video demonstrations that can be viewed on the web. ${ }^{6}$

In order to avoid a set of repetitive illustrations, the associated modem mode-switching dynamics will be exposed in more detail at a later stage, in the context of Figs. 14-17 in conjunction with our burst-by-burst adaptive CDMA system of Section IX.

In contrast to the H.261 codec, where it was legitimate to refrain from encoding specific macroblocks constituted by four $8 \times 8$-pixel video blocks, in the H.263 scheme every MB has to be transmitted. However, inactive macroblocks can be coded using a 1-bit codeword. Due to the packet drop request upon corruption now the MBs are reencoded as empty MBs, conveying no information, i.e., assuming that they contain zero-valued motion-compensated error residual pixels. This is equivalent to assuming that the video content of the current video block has not changed since the reception of the previous video frame. In such events, both the local and remote decoders have to be informed that the partially received MB has to be removed from their buffers. Hence, some parts of the picture cannot be updated during the video frame concerned. However, in most cases the dropped MBs will be updated in the next video frame. The effect of video packet dropping is not noticeable unless the video frame contains a large amount of motion and the packet dropping probability is very high. Again, these subjective effects can be viewed at various video packet dropping rates by displaying the decoded video sequences available on the Web. ${ }^{7}$ The corresponding objective PSNR degradation versus packet dropping rate is characterized by Fig. 8, indicating that as long as this dropping rate remains below about $5 \%$, the video degradation remains unobjectionable. Hence, we designed an error-rate-based power-control scheme with the specification of maintaining the required transmission packet dropping or-synonymously - transmission burst error rate, which is characterized briefly in the next section. For a full flowchart and implementational details, the interested reader is referred to [27] and [145].

\section{VIDEO PERformance USING POWER CONTROL}

In this section, we highlight the expected video system performance of the prototype design with power control, which is typically the situation in the cellular, rather than cordless, systems of Table 1, i.e., in systems, such as GSM, IS-54, IS-95, UMTS, etc.

Important contributions in the field of power control are due, e.g., to Zander [176], [177], Zorzi [178], Leung [179], Ariyavisitakul et al. [180], Pichna and Wang [181], and Lee

\footnotetext{
${ }^{6}$ http://www-mobile.ecs.soton.ac.uk/peter/robust-h263/robust.html.

${ }^{7}$ http://www-mobile.ecs.soton.ac.uk/peter/robust-h263/robust.html \#DROPPING.
}

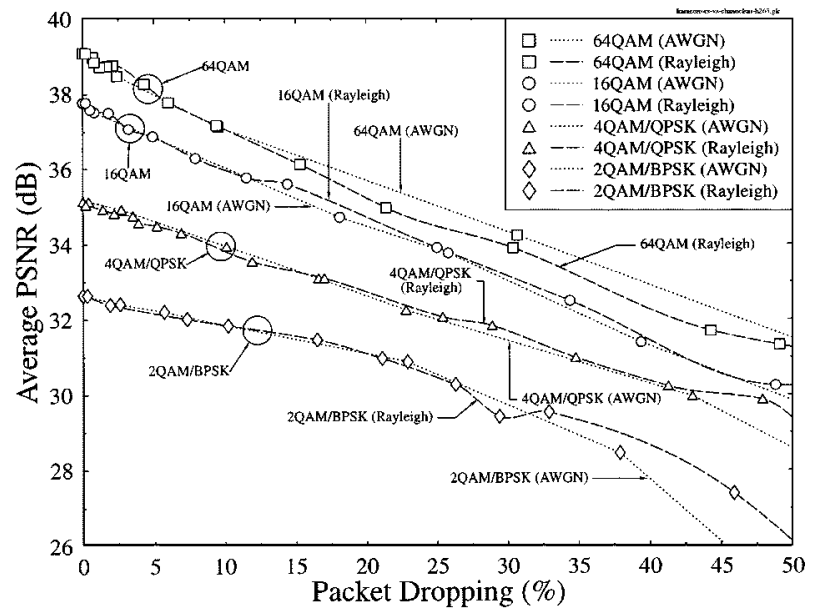

Fig. 8. Average PSNR of decoded video versus video packet dropping rate-or synonymously-transmission BurstER, for 2, 4, 16, and 64 QAM over both Gaussian and Rayleigh-fading channels. The QCIF resolution "Miss America" video sequence was used for all transmission modes (from [142]).

et al. [182]. A power-control algorithm needs an accurate and recent estimate of the radio channel's quality. The systems that rely on the received signal strength indicator (RSSI) to measure channel quality have to tolerate that the accuracy of the channel estimation is reduced in an interference-limited environment. Various techniques to reduce this inaccuracy have been suggested, in particular an averaging technique by Austin and Stüber [183]. Error-rate-based powercontrol schemes give a reliable channel quality estimation but have some limitations. An attractive power control algorithm based on a combination of BER and RSSI estimates was proposed by Chuang and Sollenberger [184]. Also Kumar et al. [185] proposed a power-control algorithm based on BER measurements.

In our proposed BER-based power-control algorithm, the main channel quality indicator was the transmission burst error flag (BEF), which we defined as a 1-bit Boolean flag, where a logical one indicated that a transmission burst was error-free while a zero signaled an erroneous transmission burst reception. In order for the transmitter to infer, whether a transmission burst was received correctly, a binary acknowledgment flag was sent from the receiver, which is associated with a delayed indication of the channel quality. This delay was explicitly quantified in Fig. 4. If this delay is too high, the transmission burst error flag may be of little use. This delay is one of the disadvantages of BER-based techniques in comparison to systems that use an RSSI reading carried out by the receiver in order to set the transmission power. However, the RSSI-based systems are easily deceived by high levels of co-channel interference.

In addition to the transmission burst error flag, which is generated by the channel decoder, our proposed power-control algorithm uses the actual number of errors corrected by the channel coding as an additional indicator of channel quality. If the number of errors in the $\mathrm{BCH}$-coded transmission burst is zero, the channel is considered good, and reducing the transmission power should be considered. By contrast, if the number of errors in the transmitted frame is 
Table 7

Power Control Algorithm's Features

\begin{tabular}{l|c}
\hline Feature & Value \\
\hline \hline Delay (TDMA frames) & 1 \\
\hline Minimum Step Size (dB) & 1 \\
\hline Maximum Step Size (dB) & 16 \\
\hline Max. TxPower (dBm) & $30(1 \mathrm{~W})$ \\
\hline Tx Power Dynamic Range (dB) & 64 \\
\hline
\end{tabular}

higher than the correcting capability of the FEC code, then a transmission frame error has occurred, and increasing the transmission power should be of urgent consideration. However, if the number of errors contained in the $\mathrm{BCH}$-coded transmission burst is correctable by the FEC, there are three possible situations that should be considered. First, if the number of errors is near to the error correction capability of the code, where a transmission burst error would occur, or the number of errors in successive transmission bursts has been increasing, then the transmission power should be increased. Secondly, if the number of errors in the transmission burst is low and has been reduced in previous transmission bursts, then the power should be reduced. Lastly, when the transmission burst is not error-free but the errors are correctable by the FEC, it is logical to keep the transmission power constant. For further intrinsic details of the power-control algorithm, interested readers are referred to [27] and [145]. The amount of time to delay an action before the power-control algorithm increases or decreases the power depends on many factors, such as the modulation scheme employed, the channel conditions, the required transmission BurstER, etc. The power-control algorithm proposed in this contribution exhibits a variable step size and has been tested with a power control delay of one TDMA frame. An advantage of this regime is that it can support the modem's operation, irrespective of the modem mode employed. Based on the above rationale, the power-control algorithm's main features are shown in Table 7. We used a typical maximum transmission power of $1 \mathrm{~W}$ with a dynamic range of $64 \mathrm{~dB}$ as in GSM.

The performance of the power-control algorithm was tested in the worst case scenario of a single interfer used to inflict co-channel interference. Examples are shown in Fig. 9(a) and (b) for the best and worst case situations using 4 QAM, where the best case is when the interferer is far from the serving BS and the user is close to its corresponding BS, and vice versa. Specifically, Fig. 9(a) shows the transmission power variation versus time, demonstrating that the transceiver is operating close to the minimum of $-34 \mathrm{dBm}$. Both of the subfigures display the slow fading, the SINR average over each time slot, the transmission burst error flag, and the transmission power. The worst case situation seen in Fig. 9(b) shows more clearly how the power control reacts to the fluctuating SINR. Observe in Fig. 9 that the transmission burst error flag suggests a moderate FEC overloading event frequency, irrespective of the path loss and slow fading experienced. Furthermore, it can also be seen that the transmission power is limited to the maximum power of $30 \mathrm{dBm}$ at one point.

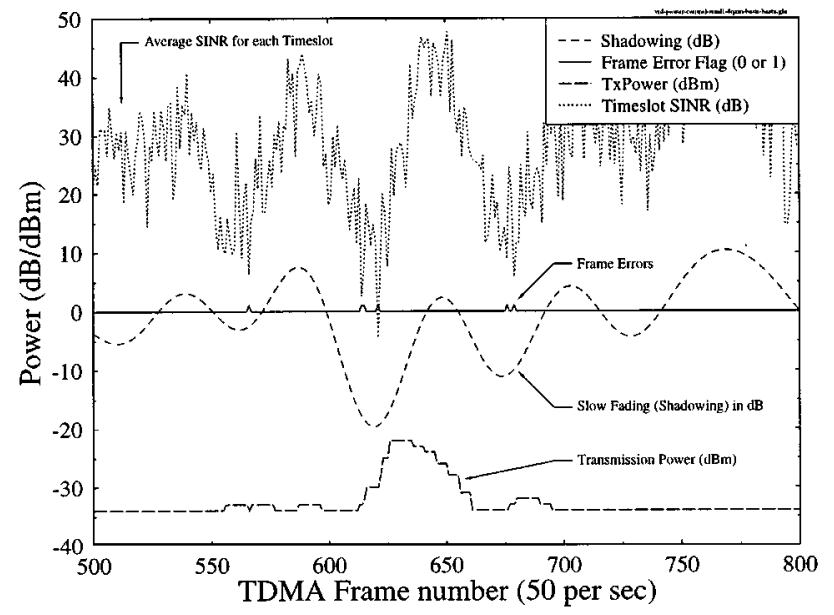

(a) Best Case

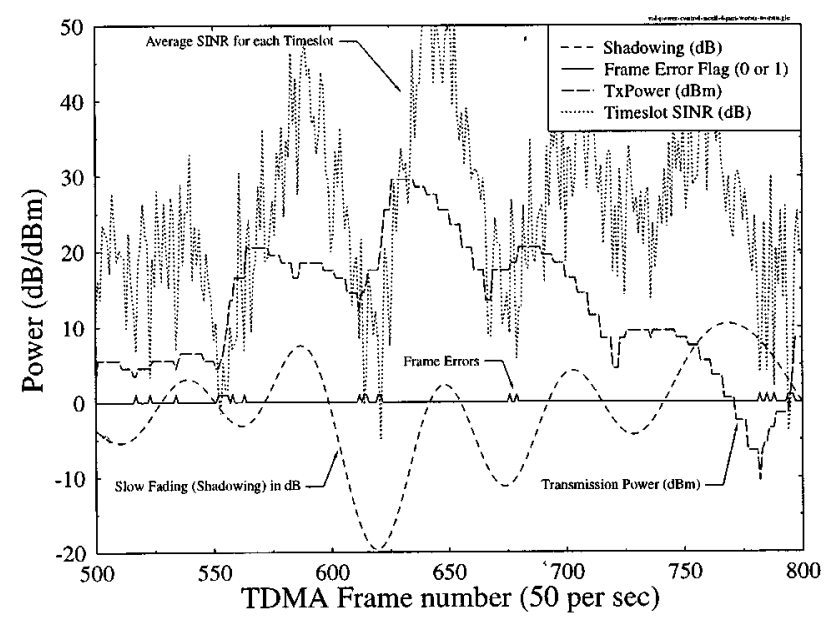

(b) Worst Case

Fig. 9. Various waveforms associated with the error rate based power control algorithm. (a) Best case situation is when both the interfer and the user are close to their BS. (b) Worst case situation is when both the interferer and the user are at the edge of their cell (from [144] and [145]).

As expected, the power-control algorithm maintains a near-constant $\mathrm{BCH}$-coded transmission burst error rate across the whole cell area, which is demonstrated by Fig. 10 . The target BurstER was adjusted to around 5\%, as required by the video transceiver, in order to maintain the target PSNR, but in the extreme vicinity of the BS the power could not be reduced below the $30-64=-34 \mathrm{dBm}$ level, which resulted in the reduced BurstER observed in Fig. 10.

An important feature of the power-control algorithm is that it is capable of maintaining the required BurstER, irrespective of the modem mode of the transceiver. Hence, it is amenable to employment in burst-by-burst adaptive video transceivers and assists in maintaining the target video quality in all modulation modes. Best performance is achieved if the power control and the modem mode switching regime are jointly optimized by ensuring that, for example, upon switching from binary phase shift 


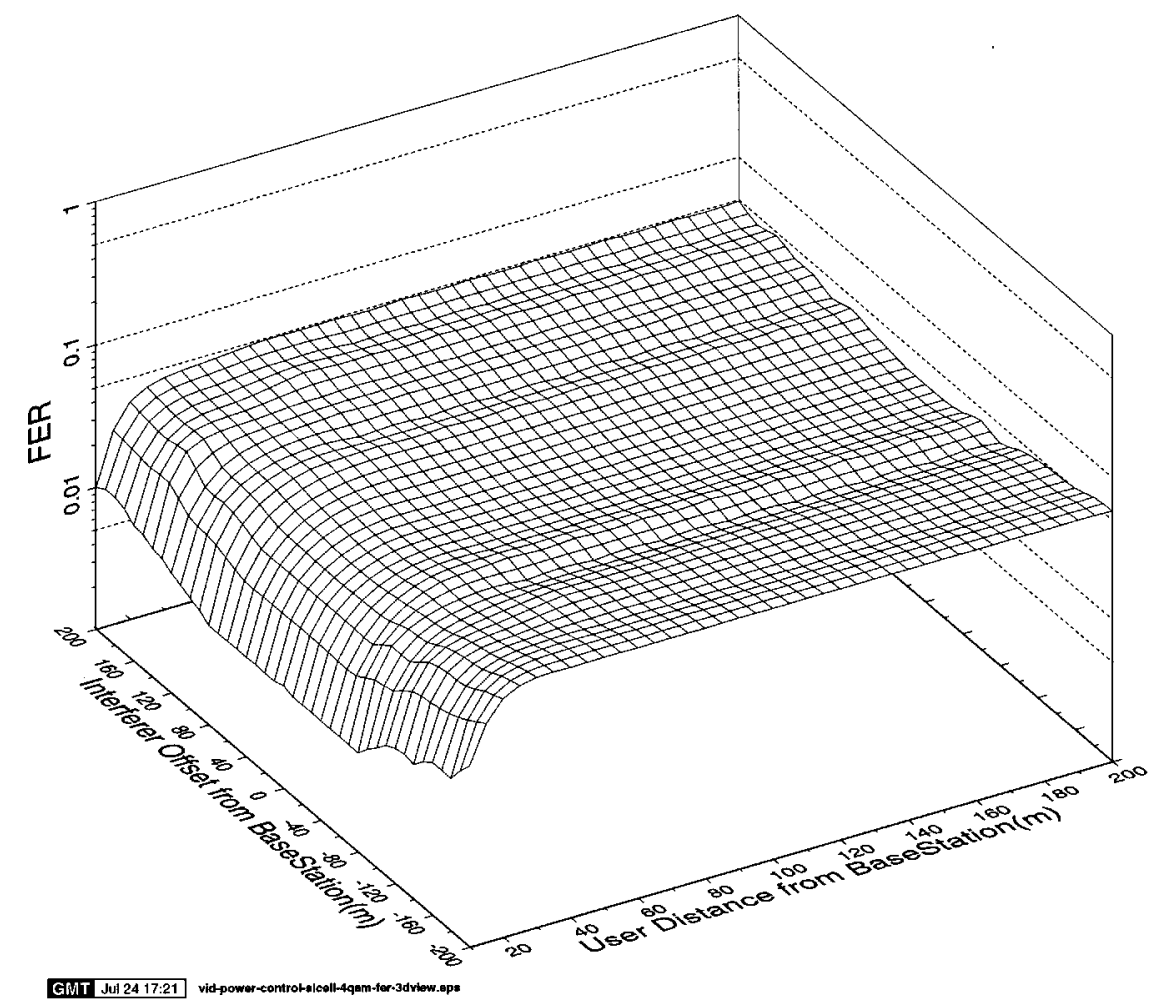

Fig. 10. Transmission BurstER with power control versus user and interferer distance from BS using 4 QAM (from [144] and [145].

keying (BPSK) to 4-QAM mode there is a slight power increase, in order to avoid the received power range near the modem mode switching thresholds. This is because without powering up, when switching from BPSK to 4 QAM the receiver would experience the lowest possible channel SNR associated with the 4-QAM mode instead of the highest possible SNR of the BPSK mode, potentially resulting in a BurstER higher than that at the top of the BPSK SNR range.

Having characterized the system architecture and the achievable performance of the TDMA/TDD-based multimode system used in our design example, let us now demonstrate in the next section how these attractive system design features can be extended to CDMA systems, which were proposed for the $3 \mathrm{G}$ wireless arrangements worldwide.

\section{BURST-BY-BURST ADAPTIVE JOINT-DETECTION CDMA VIDEO TRANSCEIVER}

\section{A. Multiuser Detection for CDMA}

The effects of multiuser interference (MAI) are similar to those of the ISI inflicted by the multipath propagation channel. More specifically, each user in a $K$-user system will suffer from MAI due to the other $(K-1)$ users. This MAI can also be viewed as a single user's signal contaminated by the ISI due to $(K-1)$ propagation paths in a multipath channel. Therefore, conventional equalization techniques used to mitigate the effects of ISI can be modified for employment in multiuser detection assisted CDMA systems. The so-called joint detection (JD) receivers constitute a category of multiuser detectors developed for synchronous burst-based CDMA transmissions, and they utilize these techniques.
Fig. 11 depicts the block diagram of a synchronous jointdetection assisted CDMA system model for uplink transmissions. There are a total of $K$ users in the system where the information is transmitted in bursts. Each user transmits $N$ data symbols per burst, and the data vector for user $k$ is represented as $\mathbf{d}^{(\mathbf{k})}$. Each data symbol is spread with a user-specific spreading sequence $\mathbf{c}^{(\mathbf{k})}$, which has a length of $Q$ chips. In the uplink, the signal of each user passes through a different mobile channel characterized by its time-varying complex impulse response $\mathbf{h}^{(\mathbf{k})}$. By sampling at the chip rate of $1 / T_{c}$, the impulse response can be represented by $W$ complex samples. Following the approach of Klein et al. [57], the received burst can be represented as $\mathbf{y}=\mathbf{A d}+\mathbf{n}$, where $\mathbf{y}$ is the received vector and consists of the synchronous sum of the transmitted signals of all the $K$ users, corrupted by a noise sequence $\mathbf{n}$. The matrix $\mathbf{A}$ is referred to as the system matrix and defines the system's response, representing the effects of MAI and the mobile channels. Each column in the matrix represents the combined impulse response obtained by convolving the spreading sequence of a user with its channel impulse response $\mathbf{b}^{(\mathbf{k})}=\mathbf{c}^{(\mathbf{k})} * \mathbf{h}^{(\mathbf{k})}$. This is the impulse response experienced by a transmitted data symbol. Upon neglecting the effects of the noise, the JD formulation is simply based on inverting the system matrix $\mathbf{A}$ in order to recover the data vector constituted by the superimposed transmitted information of all the $K$ CDMA users.

\section{B. JD-ACDMA Modem Mode Adaptation and Signaling}

In mobile communications systems, typically power-control techniques are used to mitigate the effects of path loss 


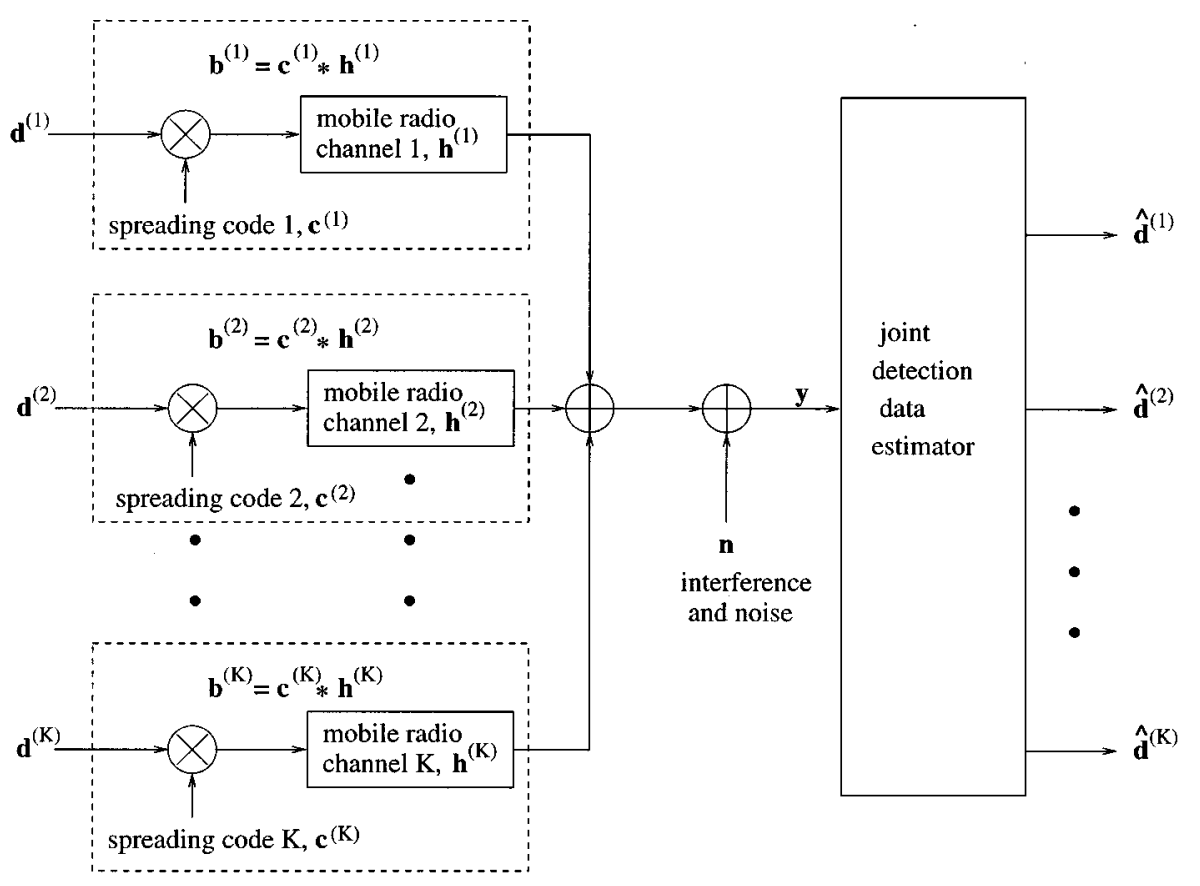

Fig. 11. System model of a synchronous CDMA system on the uplink using joint detection.

and slow fading. However, in order to counteract the problem of fast fading and co-channel interference, agile and tightspecification power-control algorithms are required [186]. Another technique that can be used to overcome the problems due to fading is adaptive-rate transmission [187], [188], where the information rate is varied according to the quality of the channel.

Different methods of multirate transmission have been proposed by Ottosson and Svensson [189]. According to the multicode method, multiple codes are assigned to a user requiring a higher bit rate [189]. Multiple data rates can also be provided by a multiple processing-gain scheme, where the chip rate is kept constant but the data rates are varied by changing the processing gain of the spreading codes assigned to the users. Performance comparisons for both of these schemes have been carried out by Ottosson $e t$ al. [189] and Ramakrishna et al. [190], demonstrating that both schemes achieved similar performance. Saquib et al. [191] and Johansson et al. [192] have also investigated the employment of the so-called decorrelating detector and the successive interference cancellation receiver for multirate CDMA systems.

Adaptive rate transmission schemes, where the transmission rate is adapted according to the channel quality, have also been proposed. Abeta et al. [193] have conducted investigations into an adaptive CDMA scheme, where the transmission rate is modified by varying the channel code rate and the processing gain of the CDMA user, employing the carrier-to-interference-and-noise ratio (CINR) as the switching metric. In their investigations, the overall packet rate was kept constant by transmitting in shorter bursts, when the transmission bit rate was high and lengthening the burst, when the bit rate was low. This resulted in a decrease in interference power, which translated to an increase in system capacity. Hashimoto et al. [194] extended this work to show that the proposed system was capable of achieving a higher capacity with a smaller handoff margin and lower average transmitter power. In these schemes, the conventional RAKE receiver was used for the detection of the data symbols. Kim [188] analyzed the performance of two different methods of combatting the mobile channel's variations, which were the adaptation of the transmitter power to compensate for channel variations or the switching of the information rate to suit the channel conditions. Using a RAKE receiver, it was demonstrated that rate adaptation provided a higher average information rate than power adaptation for a given average transmit power and a given BER.

In our design example here, we also propose to vary the information rate in accordance with the channel quality. However, in comparison to conventional power-control techniques-which may disadvantage other users in an effort to maintain the quality of the links considered-the JD-AQAM scheme employed does not disadvantage other users [29]. Additionally, burst-by-burst adaptive transceivers are capable of increasing the network capacity [195].

In this section, we will quantify the expected video performance of a range of intelligent multimode CDMA transceivers, employing JD multiuser reception CDMA techniques at the BS, which are optional in the $3 \mathrm{G}$ system proposals due to their high implementational complexity and, hence, are likely to be employed only in future implementations of the $3 \mathrm{G}$ standards. As a potential further future enhancement, we will also invoke the powerful principle of burst-by-burst adaptive JD-CDMA (JD-ACDMA) transmissions [29], which was discussed in some depth with reference to the literature in Section IV. Burst-by-burst adaptive transmissions can be readily accommodated by JD-CDMA receivers, as will be augmented in more detail 
below. The duplex JD-ACDMA video transceiver used in our system design example operates on the basis of the following philosophy.

1) The channel quality estimation is based on evaluating the mse at the output of the JD-CDMA multiuser equalizer at the receiver, as suggested for wide-band single-carrier Kalman-filtered DFE-based modems by Liew et al. in [149] and [150].

2) The decision concerning the modem mode to be used by the local transmitter for the forthcoming CDMA transmission burst is based on the prediction of the expected channel quality.

3) Specifically, if the channel quality can be considered predictable, then the channel quality estimate for the uplink can be extracted from the received signal and the receiver instructs the local transmitter as to what modem mode to use in its next transmission burst. We refer to this regime as open-loop adaptation. In this case, the transmitter has to explicitly signal the modem modes to the receiver.

4) By contrast, if the channel cannot be considered reciprocal, then the channel quality estimation is still performed at the receiver, but the receiver has to instruct the remote transmitter as to what modem modes have to be used at the transmitter in order to meet the target integrity requirements of the receiver. We refer to this mode as closed-loop adaptation.

\section{The JD-ACDMA Video Transceiver}

In this JD-CDMA system performance study, we transmitted $176 \times 144$ pixel QCIF and $128 \times 96$ pixel SQCIF video sequences at 10 frames/s using a reconfigurable TDMA/CDMA transceiver, which can be configured as a 1-, 2-, or 4-bit/symbol scheme. The H.263 video codec [27] extensively employs variable-length compression techniques and, hence, achieves a high compression ratio. However, as all entropy- and variable-length coded bit streams, its bits are extremely sensitive to transmission errors.

This error sensitivity was counteracted in our system by invoking the adaptive video packetization and video packet dropping regime of [142], when the channel codec protecting the video stream became incapable of removing all channel errors. Specifically, we refrained from decoding the corrupted video packets in order to prevent error propagation through the reconstructed video frame buffer [27], [142]. Hence-similarly to our AQAM/TDD-based system design example-these corrupted video packets were dropped at both the transmitter and receiver, and the reconstructed video frame buffer was not updated until the next video packet replenishing the specific video frame area was received. This required a low-delay, strongly protected video packet acknowledgment flag, which was superimposed on the transmitted payload packets [142]. As in the system design example of the previous section, the associated video performance degradation was found perceptually unobjectionable for transmission burst error rates below about $5 \%$.
577 microseconds

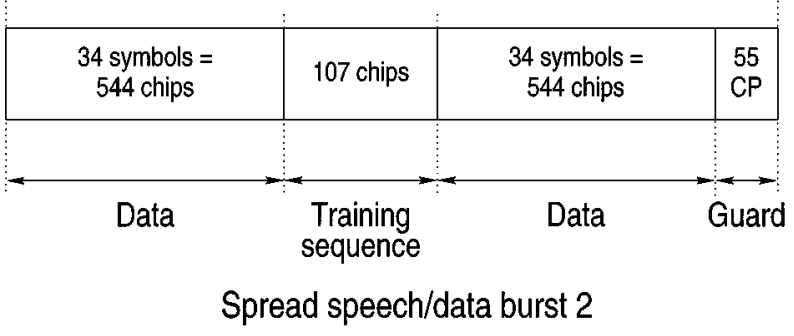

Fig. 12. Transmission burst structure of the FMA1 spread speech/data mode 2 of the FRAMES proposal [56].

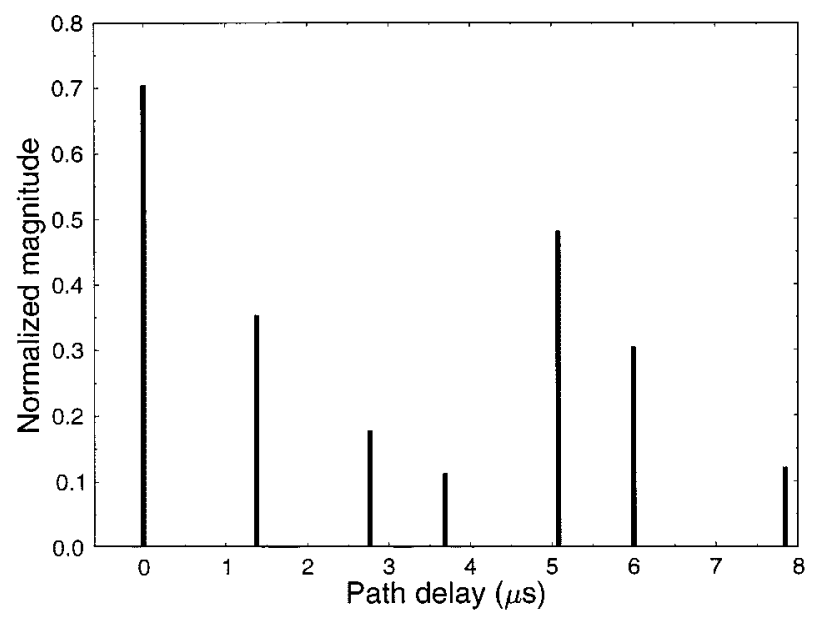

Fig. 13. Normalized channel impulse response for the COST 207 [152] seven-path bad urban channel.

The associated JD-ACDMA video system parameters are summarized in Table 8, which will be addressed in more depth during our further discourse. Employing a low spreading factor of 16 allowed us to improve the system's multiuser performance with the aid of joint-detection techniques [151] while imposing a realistic implementational complexity. This is because the JD operation is based on inverting the system matrix, which is constructed from the convolution of the channel's impulse response (CIR) and the spreading codes. Hence, maintaining a low spreading factor (SF) is critical as to the implementational complexity. We note furthermore that the implementation of the joint detection receivers is independent of the number of bits per symbol associated with the modulation mode used, since the receiver simply inverts the associated system matrix and invokes a decision concerning the received symbol, irrespective of how many bits per symbol were used. Therefore, joint detection receivers are amenable to amalgamation with the above 1-, 2-, and 4-bit/symbol CDMA modem, since they do not have to be reconfigured each time the modulation mode is switched.

In this performance study, we used the Pan-European FRAMES proposal [56] as the basis for our CDMA system. The associated transmission burst structure is shown in Fig. 12, while a range of generic system parameters are summarized in Table 8. In our performance studies, we used the COST207 [152] seven-path bad urban (BU) channel model, whose impulse response is portrayed in Fig. 13. 
Table 8

Generic System Parameters Using the FRAMES Spread Speech/Data Mode 2 Proposal [56]

\begin{tabular}{l|l}
\hline Parameter & \\
\hline \hline Multiple access & TDMA/CDMA \\
\hline Channel type & C0ST 207 Bad Urban \\
\hline Number of paths in channel & 7 \\
\hline Normalised Doppler frequency & $3.7 \times 10^{-5}$ \\
\hline CDMA spreading factor & 16 \\
\hline Spreading sequence & Random \\
\hline Tx. Frame duration & $4.615 \mathrm{~ms}$ \\
\hline Tx. Slot duration & $577 \mu \mathrm{s}$ \\
\hline Joint detection CDMA receiver & $\begin{array}{l}\text { Whitening matched filter (WMF) or Mini- } \\
\text { mum mean square error block decision feed- }\end{array}$ \\
\hline back equalizer (MMSE-BDFE) \\
\hline No. of Slots/Frame & 8 \\
\hline TDMA slots/Video packet & 3 \\
\hline Chip Periods/TDMA slot & 1250 \\
\hline Data Symbols/TDMA slot & 68 \\
\hline User Data Symbol Rate $(\mathrm{kBd})$ & 14.7 \\
\hline System Data Symbol Rate $(\mathrm{kBd})$ & 117.9 \\
\hline
\end{tabular}

Table 9

Operational-Mode Specific JD-ACDMA Video Transceiver Parameters Used in Our Design Example

\begin{tabular}{|c|c|c|c|}
\hline Features & \multicolumn{3}{|c|}{ Multi-rate System } \\
\hline Mode & BPSK & 4QAM & 16QAM \\
\hline Bits/Symbol & 1 & 2 & 4 \\
\hline FEC & \multicolumn{3}{|c|}{ Convolutional Coding } \\
\hline Octal Gen. Pol. & \multicolumn{3}{|c|}{$561 ; 753$} \\
\hline Coding-rate & \multicolumn{3}{|c|}{$R=1 / 2$} \\
\hline Constraint-length & \multicolumn{3}{|c|}{$K=9$} \\
\hline Transmitted bits/packet & 204 & 408 & 816 \\
\hline Total bit rate $(\mathrm{kbit} / \mathrm{s})$ & 14.7 & 29.5 & 58.9 \\
\hline FEC-coded bits/packet & 102 & 204 & 408 \\
\hline $\begin{array}{l}\text { Assigned to FEC-coding } \\
(\mathrm{kbit} / \mathrm{s})\end{array}$ & 7.4 & 14.7 & 29.5 \\
\hline Error detection per packet & \multicolumn{3}{|c|}{16 bit CRC } \\
\hline Feedback bits / packet & \multicolumn{3}{|c|}{9} \\
\hline Video packet size & 77 & 179 & 383 \\
\hline Packet header bits & 8 & 9 & 10 \\
\hline Video bits/packet & 69 & 170 & 373 \\
\hline $\begin{array}{l}\text { Unprotected video-rate } \\
(\mathrm{kbit} / \mathrm{s})\end{array}$ & 5.0 & 12.3 & 26.9 \\
\hline Video framerate $(\mathrm{Hz})$ & \multicolumn{3}{|c|}{10} \\
\hline
\end{tabular}

Again, the remaining generic system parameters are defined in Table 8. In our JD-ACDMA design example, we investigated the performance of a multimode convolutionally coded video system employing joint detection, while supporting two users. The associated convolutional codec parameters are summarized in Table 9 along with the operational-mode specific transceiver parameters of the multimode JD-ACDMA system. As seen in Table 9, when the channel is benign, the unprotected video bit rate will be approximately $26.9 \mathrm{~kb} / \mathrm{s}$ in the 16-QAM/JD-CDMA mode. However, as the channel quality degrades, the modem will switch to the BPSK mode of operation, where the video bit rate drops to $5 \mathrm{~kb} / \mathrm{s}$; and for maintaining a reasonable video quality, the video resolution has to be reduced to SQCIF $(128 \times 96$ pels $)$.

\section{JD-ACDMA Video Transceiver Performance}

The burst-by-burst adaptive JD-ACDMA scheme of our design example maximizes the system's throughput expressed in terms of the number of bits per transmitted nonbinary symbol by allocating the highest possible number

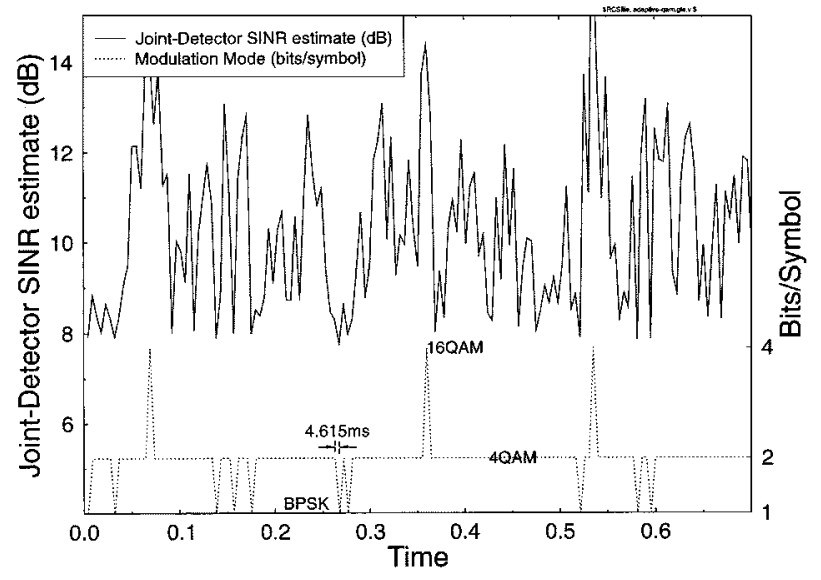

Fig. 14. Example of modem mode switching in a dynamically reconfigured burst-by-burst modem in operation, where the modulation mode switching is based upon the SINR estimate at the output of the joint detector over the channel model of Fig. 13

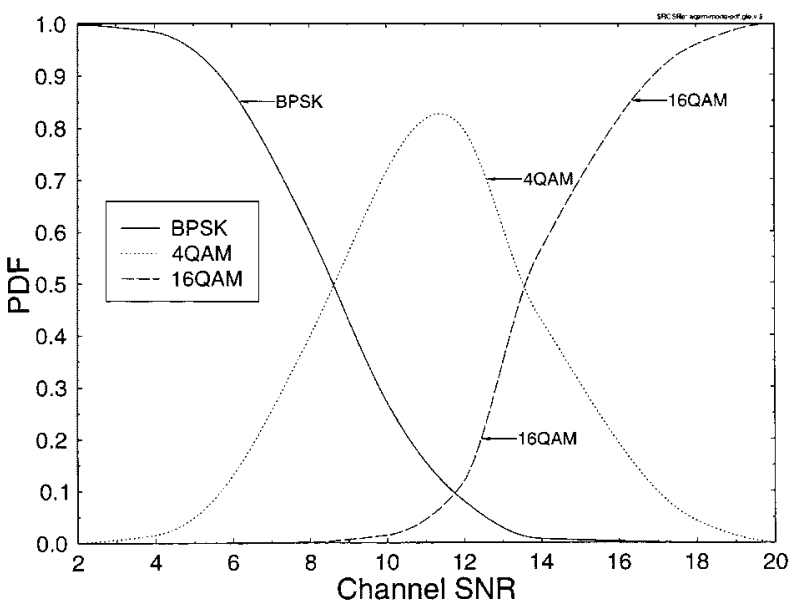

Fig. 15. PDF of the various adaptive modem modes versus channel SNR over the channel model of Fig. 13.

of bits to a symbol based on the receiver's perception concerning the instantaneous channel quality. When the instantaneous channel conditions degrade, the number of bits per symbol (BPS) is reduced, in order to maintain the required target transmission burst error rate. Fig. 14 provides a snapshot of the JD-ACDMA system's mode switching dynamics, which is based on the fluctuating channel conditions determined by all factors influencing the channel's quality, such as path loss, fast fading, slow fading, dispersion, co-channel interference, etc. The adaptive modem uses the SINR estimate at the output of the joint detector in order to estimate the instantaneous channel quality and, hence, to set the modulation mode. The probability density function (pdf) of the JD-ACDMA scheme using each modulation mode for a particular average channel SNR is portrayed in Fig. 15. It can be seen at high channel SNRs that the modem predominantly uses the 16-QAM/JD-ACDMA modulation mode, while at low channel SNRs the BPSK mode is most prevalent. However, the pdf is widely spread, indicating that often the channel quality is misjudged by the receiver due to unpredictable channel quality fluctuations caused by a high doppler frequency or co-channel interference, etc. Hence, 


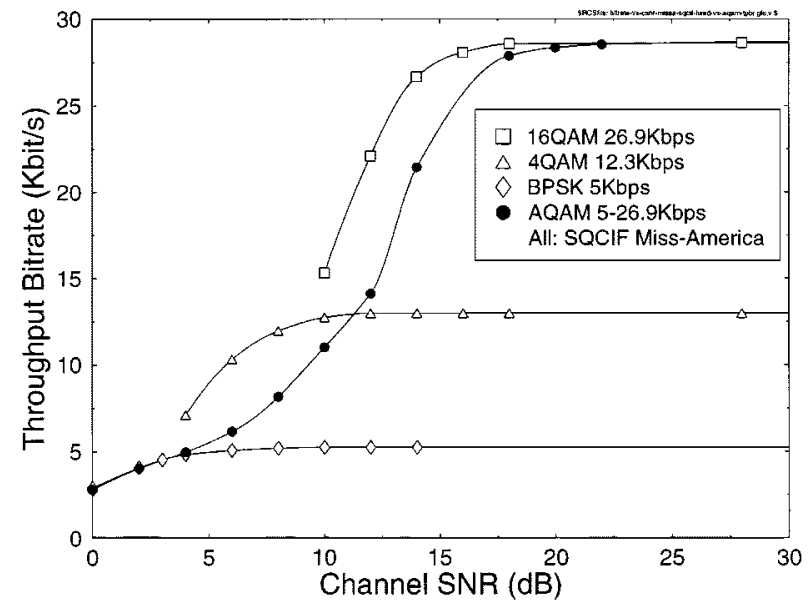

Fig. 16. Throughput bit rate versus channel SNR comparison of the three fixed modulation modes (BPSK, 4 QAM, 16 QAM) and the adaptive burst-by-burst modem (AQAM), both supporting two users with the aid of joint detection over the channel model of Fig. 13.

in certain cases, BPSK is used under high channel quality conditions or 16 QAM is employed under hostile channel conditions.

The advantage of the dynamically reconfigured burst-by-adaptive JD-ACDMA modem over a statically reconfigured system, which would be incapable of near-instantaneous channel quality estimation and modem mode switching, is that the video quality is smoothly—rather than abruptly-degraded, as the channel conditions deteriorate and vice versa. By contrast, a less "agile" statically switched or reconfigured multimode system results in more visible reductions in video quality, when the modem switches to a more robust modulation mode, as demonstrated in Fig. 16. Explicitly, Fig. 16 shows the throughput bit rate of the dynamically reconfigured burst-by-burst adaptive modem, compared to the three modes of a less agile, statically switched multimode system. The reduction of the fixed modem modes' effective throughput at low SNRs is due to the fact that under such channel conditions, an increased fraction of the transmitted packets have to be dropped, reducing the effective throughtput, since dropped packets do not contribute toward the system's effective throughput. The figure shows the smooth reduction of the throughput bit rate as the channel quality deteriorates. The burst-by-burst modem matches the BPSK mode's bit rate at low channel SNRs and the 16-QAM mode's bit rate at high SNRs. In this example, the dynamically reconfigured burst-by-burst adaptive modem characterized in the figure perfectly estimates the prevalent channel conditions, although in practice, the estimate of channel quality is not perfect and is inherently delayed. Hence, our results constitute the best case performance.

The smoothly varying effective throughput bit rate of the burst-by-burst adaptive modem translates into a smoothly varying video quality as the channel conditions change. The video quality measured in terms of the average PSNR is shown versus the channel SNR in Fig. 17 in contrast to that of the individual modem modes. The figure demonstrates

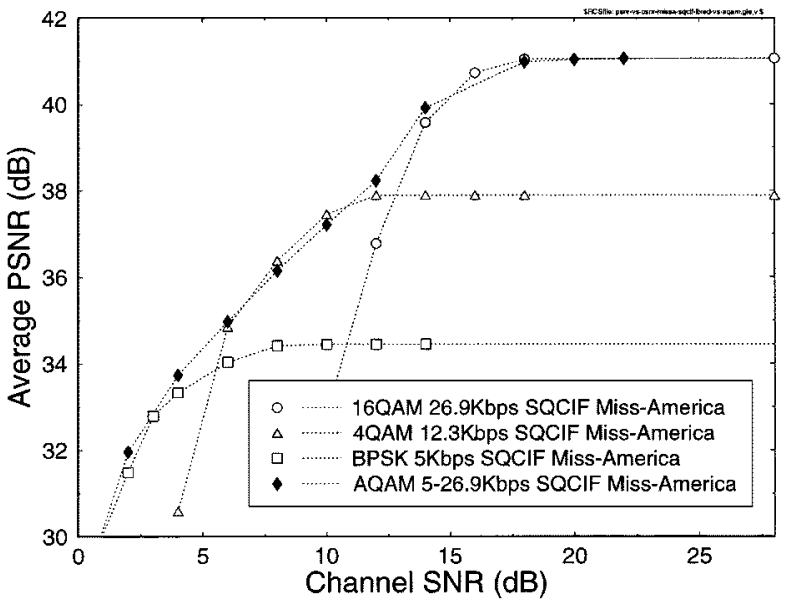

Fig. 17. Average decoded video quality (PSNR) versus channel SNR comparison of the fixed modulation modes of BPSK, 4 QAM and 16 QAM, and the burst-by-burst adaptive modem. Both support two users with the aid of joint detection. These results were recorded for the "Miss America" video sequence at SQCIF resolution $(128 \times$ 96 pels) over the channel model of Fig. 13.

that the burst-by-burst adaptive modem provides equal or better video quality over a large proportion of the SNR range shown than the individual modes. However, even at channel SNRs, where the adaptive modem has a slightly reduced PSNR, the perceived video quality of the adaptive modem is better, since the video packet loss rate is far lower than that of the fixed modem modes.

\section{SUMmarY, CONCLUSIONS, AND SYSTEM DESIGN GUIDELINES}

This paper provided a brief overview of the current $2 \mathrm{G}$ and $3 \mathrm{G}$ mobile radio scene in Section I. The cellular channel capacity was estimated across the propagation cells in Section II without power control and the geographical distribution of the SIR was analyzed in Section III. The nonuniform geographic SIR distribution and the instantaneous channel quality fluctuations due to path loss, slow fading, fast fading, co-channel interference, etc., can be exploited by designing intelligent multimode transceivers, which can be configured in more robust but lower bit-rate modes or less resilient, but high-rate, higher video quality modes, as was argued in Section IV. Section V was dedicated to an overview of the recent video compression literature and to characterizing the performance of the H.263 video codec.

On the basis of the detailed results in [27] and [142]-[147] and from our elaborations in this paper, it can be concluded that $2 \mathrm{G}$ and $3 \mathrm{G}$ interactive cordless and cellular wireless videophony using the H.263 ITU codec is realistic in a packet acknowledgment assisted TDMA/TDD or CDMA/TDD system both with and without power control following the system design guidelines of Sections VI-VIII, which are summarized as follows.

1) In time-variant wireless environments, adaptive multimode transceivers are beneficial, in particular, if the geographic distribution of the wireless channel capacity-i.e., the instantaneous channel quality- 
varies across the cell due to path loss, slow fading, fast fading, dispersion, co-channel interference, etc.

2) In order to support the operation of intelligent multimode transceivers, a video source codec is required, which is capable of conveniently exploiting the variable-rate, time-variant capacity of the wireless channel by generating always the required number of bits that can be conveyed in the current modem mode by the transmission burst. This issue was discussed in Section $\mathrm{V}-\mathrm{C}$.

3) The H.263 video codec's local and remote video reconstruction frame buffers have to be always identically updated, which requires a reliable, strongly protected acknowledgment feedback flag. The delay of this flag has to be kept as low as possible, and a convenient mechanism for its transmission is constituted by its superposition on the reverse-direction packets in TDD systems [142], although the emerging 3G CDMA systems provide also other mechanism to this effect, since their control channel rate is sufficiently high for accommodating this fast signaling requirement. The associated timing issues were summarized in Fig. 4.

4) The acknowledgment feedback flag can be conveniently error protected by simple majority logic decoded repetition codes, which typically provide more reliable protection than more complex similar-length error correction codes [147].

5) For multimode operation in cellular systems, an appropriate fixed transmission BurstER power-control algorithm is required, maintaining a constant transmission burst error rate, irrespective of the modulation mode used, since it is the BurstER, rather than the BER, that determines the associated video quality, as seen in Fig. 8 [144], [145].

6) We emphasize again that wireless video telephony over $2 \mathrm{G}$ systems is realistic using both proprietary and standard video codecs and the same design principles can also be applied in the context of the emerging $3 \mathrm{G}$ CDMA systems, as was demonstrated in the context of our JD-ACDMA design example.

7) The feasibility of using similar design principles has been shown for high-rate WATM OFDM modems in [146], [147], and [27].

Future video transceiver performance improvements are possible, while retaining the basic system design principles itemized above. The image compression community effectively completed the MPEG4 video standard [169]-[171], while the wireless communications community has commenced research toward the fourth generation of mobile radio standards. All in all, this is an exciting era for wireless video communications researchers, bringing about new standards to serve a forthcoming wireless multimedia age.

\section{ACKNOWLEDGMENT}

Over the years, the authors have had the privilege of collaborating with many former and current colleagues in the
Department of Electronics and Computer Science at the University of Southampton while working on various research projects. These colleagues and valued friends, too numerous to mention, have influenced their views concerning various aspects of wireless multimedia communications, and the authors are grateful for the enlightenment gained from their collaborations on various projects, papers, and books. They are particularly indebted to Prof. R. Steele, J. Brecht, M. Breiling, M. del Buono, C. Brooks, J. Blogh, J. Cheung, B.-J. Choi, D. Didascalou, S. Ernst, D. Greenwood, H.-T. How, T. Keller, E.-L. Kuan, C.-S. Lee, T.-H. Liew, M. Muenster, V. Roger-Marchart, R. Salami, J. Streit, J. Torrance, S. Vlahoyiannatos, W. Webb, J. Williams, J. Woodard, C.-H. Wong, H. Wong, B.-L. Yeap, M.-S. Yee, L.-L. Yang, K. Yen, A. Yuen, and many others with whom they enjoyed longer association. Their sincere thanks are also due to the EPSRC, U.K.; the Comission of the European Communities, Brussels, Belgium; Motorola ECID, Swindon, U.K.; and the Virtual Centre of Excellence (VCE) in Mobile Communications for sponsoring some of their recent research. The constructive critique of the anonymous reviewers is gratefully acknowledged.

\section{REFERENCES}

[1] W. C. Jakes, Ed., Microwave Mobile Communications. New York: Wiley, 1974.

[2] W. C. Y. Lee, Mobile Cellular Communications. New York: McGraw-Hill, 1989.

[3] _ - Mobile Communications Fundamentals. New York: Wiley, 1993.

[4] - Mobile Communications Engineering. New York: McGrawHill, 1998.

[5] J. D. Parsons and J. G. Gardiner, Mobile Communication Systems. London, U.K.: Blackie, 1989.

[6] D. Parsons, The Mobile Radio Propagation Channel. New York: Wiley/Pentech Press, 1992.

[7] K. Pahlavan and A. H. Levesque, Wireless Information Networks. New York: Wiley, 1995.

[8] K. Feher, Wireless Digital Communications, Modulation and Spread Spectrum Applications. Englewood Cliffs, NJ: Prentice-Hall, 1995.

[9] D. J. Goodman, Wireless Personal Communications Systems. Reading, MA: Addison-Wesley, 1997.

[10] R. Prasad, CDMA for Wireless Personal Communications. Norwood, MA: Artech House, 1996.

[11] T. S. Rappaport, Wireless Communications Principles and Practice. Englewood Cliffs, NJ: Prentice-Hall, 1996.

[12] V. K. Garg and J. E. Wilkes, Wireless and Personal Communications Systems. Englewood Cliffs, NJ: Prentice-Hall, 1996.

[13] J. D. Gibson, Ed., The Mobile Communications Handbook, 1st ed. Piscataway, NJ: IEEE Press/CRC Press, 1996.

[14] S. Glisic and B. Vucetic, Spread Spectrum CDMA Systems for Wireless Communications. Norwood, MA: Artech House, 1997.

[15] H. Meyr, M. Moeneclaey, and S. Fechtel, Digital Communications Receivers. New York: Wiley, 1997.

[16] S. Verdú, Multiuser Detection. Cambridge, U.K.: Cambridge Univ. Press, 1998.

[17] A. J. Viterbi, CDMA-Principles of Spread Spectrum Communication. Reading, MA: Addison-Wesley, 1995.

[18] T. Ojanperä and R. Prasad, Wideband CDMA for Third Generation Mobile Communications. Norwood, MA: Artech House, 1998.

[19] S. G. Glisic and P. A. Leppänen, Eds., Wireless Communications-TDMA Versus CDMA. Norwood, MA: Kluwer, 1997.

[20] Special Issue on the European Path Toward UMTS, IEEE Personal Commun., vol. 2, Feb. 1995.

[21] Feature Topic on, "Wireless personal communications," IEEE Personal Commun., vol. 2, Apr. 1995.

[22] Feature Topic on, "Universal telecommunications at the beginning of the 21st century," IEEE Commun. Mag., vol. 33, Nov. 1995. 
[23] Feature Topic on, "Wireless personal communications," IEEE Commun. Mag., vol. 33, Jan. 1995.

[24] Feature Topic on, "European research in mobile communications," IEEE Commun. Mag., vol. 34, pp. 60-106, Feb. 1996.

[25] V. O. K. Li and X. Qiu, "Personal communications systems," Proc. IEEE, vol. 83, pp. 1210-1243, Sept. 1995.

[26] L. Hanzo, "Bandwidth-efficient wireless multimedia communications," Proc. IEEE, vol. 86, pp. 1342-1382, July 1998.

[27] L. Hanzo, P. Cherriman, and J. Streit, Wireless Video Communications. Piscataway, NJ: IEEE Press, Dec. 2000, to be published.

[28] L. Hanzo, W. T. Webb, and T. Keller, Single- and Multi-Carrier Quadrature Amplitude Modulation. Piscataway, NJ: IEEE Press/Wiley, 2000.

[29] L. Hanzo et al., "Burst-by-burst adaptive wireless transceivers: A design paradigm for software-controlled TDMA, CDMA and OFDM Systems,". unpublished.

[30] R. Steele and L. Hanzo, Eds., Mobile Radio Communications. Piscataway, NJ: IEEE Press/Wiley, 1999.

[31] J. Streit and L. Hanzo, "Vector-quantised low-rate cordless videophone systems," IEEE Trans. Veh. Technol., vol. 42, pp. 340-357, May 1997.

[32] L. Hanzo and J. Streit, "Adaptive low-rate wireless videophone systems," IEEE Trans. Circuits Syst. Video Technol., vol. 5, pp. 305-319, Aug. 1995.

[33] J. Streit and L. Hanzo, "Quadtree-based reconfigurable cordless videophone systems," IEEE Trans. Circuits Syst. Video Technol., vol. 6, pp. 225-237, Apr. 1996.

[34] _ - "Comparative study of programmable-rate videophone codecs for existing and future multimode wireless systems," Eur. Trans. Telecommun., vol. 8, no. 6, pp. 551-572, 1997.

[35] L. Hanzo and J. Streit, "Multi-mode wireless videophony," J. Inst. Image Electron. Eng. Jpn., vol. 26, no. 6, pp. 648-659, 1997.

[36] ETSI, "Digital cellular telecommunications system (phase 2+); High speed circuit switched data (HSCSD)—Stage 1; (GSM 02.34 version 5.2.1)," Sophia Antipolis, Cedex, France, July 1997.

[37] - "Digital cellular telecommunications system (phase 2+); General packet radio service (GPRS); Overall description of the GPRS radio interface, stage 2 (GSM 03.64 version 5.2.0)," Sophia Antipolis, Cedex, France, Jan. 1998.

[38] P. Cherriman, B. L. Yeap, and L. Hanzo, "Turbo-equalised H.263based video telephony for GSM/GPRS," Globecom 2000, San Francisco, CA, Nov. 27-Dec. 1 2000, submitted for publication.

[39] L. Hanzo and J. Stefanov, "The pan-European digital cellular mobile radio system-known as GSM," in Mobile Radio Communications, R. Steele and L. Hanzo, Eds. Piscataway, NJ: IEEE Press/Wiley, 1999, ch. 8.

[40] L. Hanzo, "The pan-European mobile radio system," in The Mobile Communications Handbook, 1st ed, J. D. Gibson, Ed. Piscataway, NJ: IEEE Press/CRC Press, 1995, pp. 399-419.

[41] Mobile Station-Base Station Compatibility Standard for Dual-Mode Wideband Spread Spectrum Cellular System, EIA/TIA Interim Standard IS-95, Telecommunications Industry Association, Washington, DC, 1993.

[42] Dual-Mode Subscriber Equipment-Network Equipment Compatibility Specification, Interim Standard IS-54, Telcomm. Industry Association, Washington, DC, 1989.

[43] IS-136.1A TDMA Cellular/PCS—Radio Interface-Mobile Station-Base Station Compatibility Digital Control Channel, Revision A, Aug. 1996.

[44] TIA/EIA/IS641, Interim Standard, TDMA Cellular/PCS Radio Interface-Enhanced Full-Rate Speech Codec, May 1996.

[45] Public digital cellular (PDC) Standard, RCR STD-27, Research and Development Centre for Radio Systems, Japan.

[46] L. Hanzo, "The British cordless telephone system CT2," in The Mobile Communications Handbook, 1st ed, J. D. Gibson, Ed. Piscataway, NJ: IEEE Press/CRC Press, 1995, pp. 462-478.

[47] Research and Development Center for Radio Systems, 1993, Personal Handy Phone System, Second Generation Cordless Telephone System Standard, RCR-STD28, Tokyo, Japan, 1993.

[48] A. R. Noerpel, Y.-B. Lin, and H. Sherry, "PACS: Personal access communications system-A tutorial," IEEE Personal Commun. Mag., vol. 3, pp. 32-43, June 1996.

[49] ISO/IEC 11172 MPEG 1 International Standard, Coding of Moving Pictures and Associated Audio for Digital Storage Media Up to About $1.5 \mathrm{Mbit} / \mathrm{s}$, pt. 1-3.

[50] ISO/IEC CD 13818 MPEG 2 International Standard, Information Technology, Generic Coding of Moving Video and Associated Audio Information, pt. 1-3.
[51] ISO/IEC 14496-1: Information Technology—Coding of Audio-Visual Objects: Systems, Committee Draft, Nov. 1997.

[52] ISO/IEC 14496-2: Information Technology—Coding of Audio-Visual Objects: Visual, Committee Draft, Oct. 1997.

[53] ITU-T, "Video coding for low bit rate communication," ITU Rec. H.263, 1996.

[54] Advanced Communications Technologies and Services (ACTS), Workplan, European Commission, DGXIII-B-RA946043-WP, Aug. 1994.

[55] T. Ojanpera, "Overview of research activities for third generation mobile communications," in Wireless Communications-TDMA Versus CDMA, S. G. Glisic and P. A. Leppänen, Eds. Norwell, MA: Kluwer, 1997, pp. 415-446.

[56] A. Klein et al., "FRAMES multiple access mode 1-Wideband TDMA with and without spreading," in Proc 7th Personal, Indoor and Mobile Radio Communications (PIMRC) Conf., Helsinki, Finland, Sept. 1997, pp. 37-41.

[57] A. Klein and P. W. Baier, "Linear unbiased data estimation in mobile radio systems applying CDMA," IEEE J. Select. Areas Commun., vol. 11, pp. 1058-1066, Sept. 1993.

[58] C. Berrou, A. Glavieux, and P. Thitimajshima, "Near Shannon limit error-correcting coding and decoding, turbo-codes," in Proc. Int. Commun. Conf. (ICC 1993), pp. 1064-1070.

[59] C. Berrou and A. Glavieux, "Near optimum error correcting coding and decoding, turbo-codes," IEEE Trans. Commun., vol. 44, no. 10, pp. 1261-1271, 1996.

[60] A. Toskala, J. P. Castro, E. Dahlman, M. Latva-aho, and T. Ojanperä, "FRAMES FMA2 wideband-CDMA for UMTS," Eur. Trans. Telecommun., vol. 9, pp. 325-336, July-Aug. 1998.

[61] E. Dahlman, B. Gudmundson, M. Nilsson, and J. Sköld, "UMTS/IMT-2000 based on wideband CDMA," IEEE Commun. Mag., vol. 36, pp. 70-80, Sept. 1998.

[62] T. Ojanperä, "Overview of research activities for third generation mobile communication," in Wireless Communications TDMA versus CDMA, S. G. Glisic and P. A. Leppänen, Eds. Norwell, MA: Kluwer, 1997, pp. 415-446.

[63] ETSI/SMG/SMG2, "The ETSI UMTS terrestrial radio access (UTRA) ITU-R RTT candidate submission,” Tech. Rep., June 1998.

[64] ARIB, "Japan's proposal for candidate radio transmission technology on IMT-2000: W-CDMA," Tech. Rep., Association of Radio Industries and Businesses, June 1998.

[65] F. Adachi, M. Sawahashi, and H. Suda, "Wideband DS-CDMA for next-generation mobile communications systems," IEEE Commun. Mag., vol. 36, pp. 56-69, Sept. 1998.

[66] F. Adachi and M. Sawahashi, "Wideband wireless access based on DS-CDMA," IEICE Trans. Commun., vol. E81-B, pp. 1305-1316, July 1998.

[67] A. Sasaki, "Current situation of IMT-2000 radio transmission technology study in Japan," IEICE Trans. Commun., vol. E81-B, pp. 1299-1304, July 1998.

[68] K. Yen and L. Hanzo, "Third-generation CDMA systems," in Wireless Video Communications: Second to third generation and beyond. Piscataway, NJ: IEEE Press, Dec. 2000, to be published.

[69] E. L. Kuan, C. H. Wong, and L. Hanzo, "Burst-by-burst adaptive joint detection CDMA," in Proc. Vehicular Technology Conf. (VTC'99), Houston, TX, May 1999, pp. 1628-1632.

[70] D. N. Knisely, S. Kumar, S. Laha, and S. Nanda, "Evolution of wireless data services-IS-95 to cdma2000," IEEE Commun. Mag., vol. 36, pp. 140-149, Oct. 1998.

[71] TIA, "The cdma2000 ITU-R RTT candidate submission," Tech. Rep., 1998.

[72] D. N. Knisely, Q. Li, and N. S. Rames, "cdma2000—A third generation radio transmission technology," Bell Labs Tech. J., vol. 3, pp. 63-78, July-Sept. 1998.

[73] Y. Okumura and F. Adachi, "Variable-rate data transmission with blind rate detection for coherent DS-CDMA mobile radio," IEICE Trans. Commun., vol. E81B, pp. 1365-1373, July 1998.

[74] T. Ojanperä and R. Prasad, "An overview of air interface multiple access for IMT-2000/UMTS," IEEE Commun. Mag., vol. 36, pp. 82-95, Sept. 1998.

[75] S. Nanda, K. Balachandran, and S. Kumar, "Adaptation techniques in wireless packet data services," IEEE Commun. Mag., vol. 38, pp. 54-64, Jan. 2000.

[76] W. C. Y. Lee, "Estimate of channel capacity in Rayleigh fading environment," IEEE Trans. Veh. Technol., vol. 39, pp. 187-189, Aug. 1990. 
[77] C. C. Lee and R. Steele, "Signal-to-interference calculations for modern TDMA cellular communication systems," Proc. Inst. Elect. Eng. Commun., vol. 142, pp. 21-30, Feb. 1995.

[78] J. E. B. Williams, L. Hanzo, R. Steele, and J. C. S. Cheung, "A comparative study of microcellular speech transmission schemes," IEEE Trans. Veh. Technol., vol. 43, pp. 909-924, Nov. 1994.

[79] J. E. B. Williams, L. Hanzo, and R. Steele, "Channel-adaptive voice communications," in Proc. Inst. Elect. Eng. Radio Receivers and Associated Systems (RRAS'95) Conf., Conf. Public 415, Bath, U.K., Sept. 26-28, 1995, pp. 144-147.

[80] R. Steele and W. T. Webb, "Variable rate QAM for data transmission over Rayleigh fading channels," in Proc. Wireless'91. Calgary, Alberta, 1991, pp. 1-14.

[81] W. Webb and R. Steele, "Variable rate QAM for mobile radio," IEEE Trans. Commun., vol. 43, no. 7, pp. 2223-2230, 1995.

[82] S.-G. Chua and A. Goldsmith, "Variable-rate variable-power mqam for fading channels," in Proc. IEEE 46th Vehicular Technology Conf., 1996, pp. 815-819.

[83] M.-S. Alouini and A. Goldsmith, "Area spectral efficiency of cellular mobile radio systems," in Proc. IEEE Vehicular Technology Conf. (VTC'97), Phoenix, AZ, pp. 652-656.

[84] - "Capacity of Nakagami multipath fading channels," in Proc. IEEE Vehicular Technology Conf. (VTC'97), Phoenix, AZ, pp. 652-656.

[85] A. Goldsmith, "The capacity of downlink fading channels with variable rate and power," IEEE Trans. Veh. Technol., vol. 46, pp. 569-580, Aug. 1997.

[86] Y. Kamio, S. Sampei, H. Sasaoka, and N. Morinaga, "Performance of modulation-level-control adaptive-modulation under limited transmission delay time for land mobile communications," in Proc. IEEE 45th Vehicular Technology Conf., 1995, pp. 221-225.

[87] S. Sampei, S. Komaki, and N. Morinaga, "Adaptive modulation/TDMA scheme for large capacity personal multi-media communication systems," IEICE Trans. Commun., vol. 77, no. 9, pp. 1096-1103, 1994.

[88] M. Morimoto, H. Harada, M. Okada, and S. Komaki, "A study on power assignment of hierarchical modulation schemes for digital broadcasting," IEICE Trans. Commun., vol. 77, no. 12, pp. 1495-1500, 1994.

[89] S. Otsuki, S. Sampei, and N. Morinaga, "Square-QAM adaptive modulation TDMA/TDD systems using modulation level estimation with Walsh function," Electron. Lett., pp. 169-171, Nov. 1995.

[90] D. A. Pearce, A. G. Burr, and T. C. Tozer, "Comparison of countermeasures against slow Rayleigh fading for TDMA systems," in Inst. Elect. Eng. Colloquium Advanced TDMA Techniques and Applications, London, U.K., 1996, pp. 9/1-9/6.

[91] J. M. Torrance and L. Hanzo, "Upper bound performance of adaptive modulation in a slow Rayleigh fading channel," Electron. Lett., pp. 169-171, Apr. 1996.

[92] — "Adaptive modulation in a slow Rayleigh fading channel," in Proc. IEEE 7th Personal, Indoor and Mobile Radio Communications (PIMRC) Conf., 1996, pp. 497-501.

[93] _ " "Optimisation of switching levels for adaptive modulation in a slow Rayleigh fading channel,” Electron. Lett., pp. 1167-1169, June 1996.

[94] - "Demodulation level selection in adaptive modulation," Electron. Lett., vol. 32, pp. 1751-1752, Sept. 12, 1996.

[95] — , "Latency considerations for adaptive modulation in slow Rayleigh fading," in Proc. IEEE Vehicular Technology Conf. (VTC'97), Phoenix, AZ, 1997, pp. 1204-1209.

[96] — "Latency and networking aspects of adaptive modems over slow indoors Rayleigh fading channels," IEEE Trans. Veh. Technol., vol. 48, pp. 1237-1251, July 1999.

[97] _ - "Interference aspects of adaptive modems over slow Rayleigh fading channels," IEEE Trans. Veh. Technol., vol. 48, pp. 1527-1545, Sept. 1999.

[98] — "Statistical multiplexing for mitigating latency in adaptive modems," in Proc. 7th Personal, Indoor and Mobile Radio Communications (PIMRC) Conf., Helsinki, Finland, Sept. 1-4, 1997, pp. $938-942$.

[99] C. H. Wong and L. Hanzo, "Upper-bound performance of a wideband burst-by-burst adaptive modem," IEEE Trans. Commun., vol. 48, pp. 367-369, Mar. 2000.

[100] K. R. Narayanan and L. J. Cimini, Jr., "Equalizer adaptation algorithms for high speed wireless communications," in Proc. IEEE Vehicular Technology Conf. (VTC'96), 1997, pp. 681-685.
[101] N. Jayant and P. Noll, Digital Coding of Waveforms, Principles and Applications to Speech and Video. Englewood Cliffs, NJ: PrenticeHall, 1984.

[102] R. J. Clarke, Transform Coding of Images. New York: Academic, 1985.

[103] A. Netravali and B. Haskell, Digital Pictures: Representation and Compression. New York: Plenum, 1988.

[104] B. G. Haskell, A. Puri, and A. N. Netravali, Digital Video, an Introduction to MPEG-2. New York: Chapman \& Hall, 1997.

[105] K. Jack, Video Demystified. San Diego, CA: Hightext Publications, 1993.

[106] A. K. Jain, Fundamentals of Digital Image Processing. Englewood Cliffs, NJ: Prentice-Hall, 1989.

[107] K. Rao and P. Yip, Discrete Cosine Transform. New York: Academic, 1990.

[108] A. M. Tekalp, Digital Video Processing. Englewood Cliffs, NJ: Prentice-Hall, 1995.

[109] H. Gharavi, H. Yasuda, and T. Meng, Eds., IEEE Trans. Circuits Syst. Video Technol. (Special Issue on Visual Communications), Apr. 1996, vol. 6.

[110] H. Gharavi, Ed., Image Commun. (Special Issue on Signal Processing), 1998, vol. 12.

[111] H. Gharavi and L. Hanzo, Proc. IEEE (Special Issue on Video Transmission for Mobile Multimedia Applications), vol. 87, pp. 1703-1848, Oct. 1999.

[112] B. Girod and N. Färber, "Feedback-based error control for mobile video transmission," Proc. IEEE, vol. 87, pp. 1707-1723, Oct. 1999.

[113] J. D. Villasenor, Y.-Q. Zhang, and J. Wen, "Robust video coding algorithms and systems," Proc. IEEE, vol. 87, pp. 1724-1750, Oct. 1999.

[114] R. E. Van Dyck and D. J. Miller, "Transport of wireless video using separate, concatenated and joint source-channel coding," Proc. IEEE, vol. 87, pp. 1751-1763, Oct. 1999.

[115] H. Gharavi and S. M. Alamouti, "Multipriority video transmission for third-generation wireless communication systems," Proc. IEEE, vol. 87, pp. 1764-1777, Oct. 1999.

[116] H. Rohling, T. May, K. Brüninghaus, and R. Grünheid, "Broadband OFDM radio transmission for multimedia applications," Proc. IEEE, vol. 87, pp. 1778-1789, Oct. 1999.

[117] D. Raychaudhuri, "Wireless ATM networks: Technology status and future directions," Proc. IEEE, vol. 87, pp. 1790-1806, Oct. 1999.

[118] P.-R. Chang and C.-F. Lin, "Wireless ATM-based multicode CDMA transport architecture for MPEG2 video transmission," Proc. IEEE, vol. 87, pp. 1807-1828, Oct. 1999.

[119] K. H. Tzou, H. G. Mussmann, and K. Aizawa, Eds., IEEE Trans. Circuits Syst. Video Technol. (Special Issue on Very Low Bit Rate Video Coding), June 1994, vol. 4, pp. 213-357.

[120] N. Hubing, Ed., "Speech and image coding," in IEEE J. Select. Areas Commun., June 1992, vol. 10, pp. 793-976.

[121] B. Girod et al., Ed., IEEE Trans. Image Compression (Special Issue on Image Sequence Compression), Sept. 1994, vol. 3, pp. 465-716.

[122] M. F. Chowdhury et al., "A switched model-based coder for video signals," IEEE Trans. Circuits Syst. Video Technol., vol. 4, pp. 228-235, June 1994.

[123] G. Bozdagi et al., "3-D motion estimation and wireframe adaptation including photometric effects for model-based coding of facial image sequences," IEEE Trans. Circuits Syst. Video Technol., vol. 4, pp. 246-256, June 1994.

[124] C. S. Choi et al., "Analysis and synthesis of facial image sequences in model-based image coding," IEEE Trans. Circuits Syst. Video Technol., vol. 4, pp. 257-275, June 1994.

[125] R. Stedman, H. Gharavi, L. Hanzo, and R. Steele, "Transmission of subband-coded images via mobile channels," IEEE Trans. Circuits Syst. Video Technol., vol. 3, pp. 15-27, Feb. 1993.

[126] J. W. Woods, Ed., Subband Image Coding. Norwell, MA: Kluwer, 1991.

[127] H. Gharavi, "Subband coding of video signals," in Subband Image Coding, J. W. Woods, Ed. Norwell, MA: Kluwer, 1991, ch. 6, pp. 229-271.

[128] K. N. Ngan and W. L. Chooi, "Very low bit rate video coding using 3D subband approach," IEEE Trans. Circuits Syst. Video Technol., vol. 4, pp. 309-316, June 1994.

[129] L. Hanzo and J. Streit, "A fractal video communicator," in Proc. IEEE Vehicular Technology Conf. (VTC'94), Stockholm, Sweden, June 7-11, pp. 1030-1034. 
[130] M. Khansari, A. Jalali, E. Dubois, and P. Mermelstein, "Robust low bit-rate video transmission over wireless access systems," in Proc. Int. Communications Conf. (ICC), 1994, pp. 571-575.

[131] N. Färber, E. Steinbach, and B. Girod, "Robust H.263 video transmission over wireless channels," in Proc. Int. Picture Coding Symp. (PCS), Melbourne, Australia, Mar. 1996, pp. 575-578.

[132] E. Steinbach, N. Färber, and B. Girod, "Standard compatible extension of H.263 for robust video transmission in mobile environments," IEEE Trans. Circuits Syst. Video Technol., vol. 7, no. 6, pp. 872-881, 1997.

[133] B. Girod, E. Steinbach, and N. Färber, "Performance of the H.263 video compression standard," J. VLSI Signal Processing (Special Issue on Recent Development in Video), vol. 17, no. 2-3, pp. 101-111, 1997.

[134] B. Girod, N. Färber, and E. Steinbach, "Standards based video communication at very low bit-rates," presented at the Proc. VIII Eur. Signal Processing Conf. (EUSIPCO-96), Trieste, Italy, Sept. 10-13, 1996.

[135] N. Färber, E. Steinbach, and B. Girod, "Robust H.263 compatible transmission for mobile video server access," in Proc. 1st Int. Workshop Wireless Image/Video Communications, Loughborough, U.K., Sept. 4-5, 1996, pp. 8-13.

[136] E. Steinbach, A. Hanjalic, and B. Girod, "3D motion and scene structure estimation with motion dependent distortion of measurement windows," in Proc. ICIP-96, vol. 1, Lausanne, Switzerland, Sept. 16-19, pp. 61-68.

[137] M. Horn and B. Girod, "Performance analysis of multiscale motion compensation techniques in pyramid coders," in Proc. ICIP-96, vol. 3, Lausanne, Switzerland, Sept. 16-19, pp. 255-258.

[138] F. Eryurtlu, A. H. Sadka, and A. M. Kondoz, "Error robustness improvement of video codecs with two-way decodable codes," Electron. Lett., vol. 33, pp. 41-43, Jan. 2, 1997.

[139] A. H. Sadka, F. Eryurtlu, and A. M. Kondoz, "Improved performance H.263 under erroneous transmission conditions," Electron. Lett., vol. 33, pp. 122-124, Jan. 16, 1997.

[140] K. N. Ngan and D. Chai, "Very low bit rate video coding using H.263 coder," IEEE Trans. Circuits Syst. Video Technol., vol. 6, pp. 308-312, June 1996.

[141] K. H. H. Wong and L. Hanzo, "Channel coding," in Mobile Radio Communications, R. Steele and L. Hanzo, Eds. Piscataway, NJ: IEEE Press/Wiley, 1999, ch. 4, pp. 347-488.

[142] P. Cherriman and L. Hanzo, "Programmable H.263-based wireless video transceivers for interference-limited environments," IEEE Trans. Circuits Syst. Video Technol., vol. 8, pp. 275-286, June 1998.

[143] S. Lin, D. J. Costello, and M. J. Miller, "Automatic-repeat-request error-control schemes," IEEE Commun. Mag., pp. 5-17, Dec. 1984.

[144] P. Cherriman and L. Hanzo, "H.261 and H.263 wireless videophone performance in iterference-limited scenarios," in Proc. 6th Personal, Indoor and Mobile Radio Communications (PIMRC) Conf., Taipei, Taiwan, Oct. 15-18, 1996, pp. 158-162.

[145] — , "Power-controlled multimode video telephony," IEEE Trans. Veh. Technol., vol. 48, pp. 1726-1738, Sept. 1999.

[146] T. Keller, P. Cherriman, and L. Hanzo, "Orthogonal frequency division multiplex transmission of H.263 encoded video over wireless ATM networks," in Proc. Advanced Communications Technologies and Services (ACTS) Summit'97, Aalborg, Denmark, Oct. 1997, pp. 276-281

[147] P. Cherriman, T. Keller, and L. Hanzo, "Orthogonal frequency division multiplex transmission of H.263 encoded video over highly frequency-selective wireless networks," IEEE Trans. Circuits Syst. Video Technol., vol. 9, pp. 701-712, Aug. 1999.

[148] T. Keller and L. Hanzo, "Adaptive multicarrier modulation: a convenient framework for time-frequency processing in wireless communications," Proc. IEEE, vol. 88, pp. 611-640, May 2000.

[149] T. H. Liew, C. H. Wong, and L. Hanzo, "Block turbo coded burst-byburst adaptive modems," in Proc. Microcoll'99, Budapest, Hungary, Mar. 21-24, 1999, pp. 59-62.

[150] C. H. Wong, T. H. Liew, and L. Hanzo, "Blind modem mode detection aided block turbo coded burst-by-burst wideband adaptive modulation," in Proc. Advanced Communications Technologies and Services (ACTS) Summit'99, Sorrento, Italy, June 8-11, 1999. Paper 10254.

[151] E. L. Kuan and L. Hanzo, "Joint detection CDMA techniques for third-generation transceivers," in Proc. Advanced Communications Technologies and Services (ACTS) Summit'98, Rhodes, Greece, June 8-11, 1998, pp. 727-732.
[152] "COST 207, Digital land mobile radio communications, final report," Office for Official Publications of the European Communities, Luxembourg, 1989.

[153] J. F. A. Magarey and N. G. Kingsbury, "Motion estimation using complex wavelets," in Proc. Int. Conf. Acoustics Speech and Signal Processing (ICASSP96), vol. IV, Atlanta, GA, May 1996, pp. 2371-2374.

[154] P. J. Czerepinski and D. R. Bull, "Coder-oriented matching criteria for motion estimation," in Proc 1st Int. Workshop Wireless Image/Video Communications, U.K.: Loughborough Univ., Sept. 4-5, 1996, pp. 38-42.

[155] J. Nieweglowski, T. Moisala, and P. Haavisto, "Motion compensated video sequence interpolation using digital image warping," in Proc. IEEE Int. Conf. Acoustics Speech and Signal Processing (ICASSP'94), vol. 5, 1994, pp. 5-205-5-208.

[156] D. W. Redmill and N. G. Kingsbury, "Improving the error resilience of entropy encoded video signals," in Proc. Conf. Image Processing: Theory and Applications (IPTA), 1993, pp. 67-70.

[157] D. Raychaudhuri and N. Wilson, "Multimedia personal communication networks, system design issues," in Proc. 3rd WINLAB Workshop 3rd Generation Wireless Information Networks, Apr. 1992, pp. 259-288.

[158] — "ATM based transport architecture for multiservices wireless personal communication network," in IEEE J. Select. Areas Commun., Oct. 1994, pp. 1401-1414.

[159] C.-K. Toh, Wireless ATM and Ad Hoc Networks, Protocols and Architectures. Norwell, MA: Kluwer, 1997.

[160] J. Brecht, L. Hanzo, and M. Del Buono, "Multi-frame packet reservation multiple access for variable-rate users," in Proc. 7th Personal, Indoor and Mobile Radio Communications (PIMRC) Conf., Helsinki, Finland, Sept. 1-4, 1997, pp. 430-438.

[161] J. Brecht, M. Del Buono, and L. Hanzo, "Multi-frame packet reservation multiple access using oscillation-scaled histogram-based Markov modeling of video codecs," Image Commun., vol. 12, pp. 167-182, 1998.

[162] J. Brecht and L. Hanzo, "Statistical packet assignment multiple access for wireless asynchronous transfer mode systems," in Proc. Advanced Communications Technologies and Services (ACTS) Summit'97, Aalborg, Denmark, Oct. 1997, pp. 734-738.

[163] R. M. Pelz, "An unequal error protected $p \times 8 \mathrm{kbit} / \mathrm{s}$ video transmission for DECT," in Proc. IEEE Vehicular Technology Conf., 1994, pp. 1020-1024.

[164] T. Chen, "A real-time software based end-to-end wireless visual communications simulation platform," in Proc. SPIE Conf. Visual Communications and Image Processing, 1995, pp. 1068-1074.

[165] K. Illgner and D. Lappe, "Mobile multimedia communications in a universal telecommunications network," in Proc. SPIE Conf. Visual Communications and Image Processing, 1995, pp. 1034-1043.

[166] Y. Zhang, "Very low bit rate video coding standards," in Proc. SPIE Conf. Visual Communications and Image Processing, 1995, pp. 1016-1023.

[167] H. Ibaraki et al., "Mobile video communication techniques and services," in Proc. SPIE Conf. Visual Communications and Image Processing, 1995, pp. 1024-1033.

[168] K. Watanabe et al., "A study on transmission of low bit-rate coded video over radio links," in Proc. SPIE Conf. Visual Communications and Image Processing, 1995, pp. 1025-1029.

[169] Y.-Q. Zhang, F. Pereira, T. Sikora, and C. Reader, Eds., IEEE Trans. Circuits Syst. Video Technol. (Special Issue on MPEG4), Feb. 1997, vol. 7, pp. 1-256.

[170] L. Chiariglione, "MPEG and multimedia communication," IEEE Trans. Circuits Syst. Video Technol., vol. 7, pp. 5-18, Feb. 1997.

[171] T. Sikora, "The MPEG-4 video standard verification model," IEEE Trans. Circuits Syst. Video Technol., vol. 7, pp. 19-31, Feb. 1997.

[172] A. Gersho and R. M. Gray, Vector Quantization and Signal Compression. Norwell, MA: Kluwer, 1992.

[173] B. Ramamurthy and A. Gersho, "Classified vector quantization of images," IEEE Trans. Commun., vol. C-31, pp. 1105-1115, Nov. 1986.

[174] L. Torres and J. Huguet, “An improvement on codebook search for vector quantization,” IEEE Trans. Commun., vol. 42, pp. 208-210, Feb. 1994.

[175] M. W. Whybray and W. Ellis, "H.263-Video coding recommendation for PSTN videophone and multimedia," in Inst. Elect. Eng. Colloquium Dig., London, U.K., June 1995, pp. 6/1-6/9. 
[176] J. Zander, "Performance of optimum transmitter power control in cellular radio systems," IEEE Trans. Veh. Technol., vol. 41, pp. 57-62, Feb. 1992.

[177] — "Distributed cochannel interference control in cellular radio systems," IEEE Trans. Veh. Technol., vol. 41, pp. 305-311, Aug. 1992.

[178] M. Zorzi, "Power control and diversity in mobile radio cellular systems in the presence of ricean fading and log-normal shadowing," IEEE Trans. Veh. Technol., vol. 45, pp. 373-382, May 1996.

[179] Y.-W. Leung, "Power control in cellular networks subject to measurement error," IEEE Trans. Commun., vol. 44, pp. 772-775, July 1996.

[180] S. Ariyavisitakul and L. F. Chang, "Signal and interference statistics of a CDMA system with feedback power control," IEEE Trans. Commun., vol. 41, pp. 1626-1634, Nov. 1993.

[181] R. Pichna and Q. Wang, "Power control," in The Mobile Communications Handbook, 1st ed, J. D. Gibson, Ed. Piscataway, NJ: CRC Press/IEEE Press, 1996, ch. 23, pp. 370-380.

[182] T.-H. Lee, J.-C. Lin, and Y. T. Su, "Downlink power control algorithms for cellular radio systems," IEEE Trans. Veh. Technol., vol. 44, pp. 89-94, Feb. 1995.

[183] M. D. Austin and G. L. Stüber, "In-service signal quality estimation for TDMA cellular systems," in Wireless Personal Communications. Norwell, MA: Kluwer, 1995, vol. 2, pp. 245-254.

[184] J. C. I. Chuang and N. R. Sollenberger, "Uplink power control for TDMA portable radio channels," IEEE Trans. Veh. Technol., vol. 43, pp. 33-39, Feb. 1994.

[185] P. S. Kumar, R. D. Yates, and J. Holtzman, "Power control based on bit error rate (ber) measurements," presented at the Proc. Military Communications Conf. (MILCOM), San Diego, CA, Nov. 5-8, 1995.

[186] K. S. Gilhousen, I. M. Jacobs, R. Padovani, A. J. Viterbi, L. A. Weaver, and C. E. Wheatley, "On the capacity of a cellular CDMA system design,” IEEE Trans. Veh. Technol., vol. 40, pp. 303-312, May 1991.

[187] W. T. Webb and R. Steele, "Variable rate QAM for mobile radio," IEEE Trans. Commun., vol. 43, pp. 2223-2230, July 1995.

[188] S. W. Kim, "Adaptive rate and power DS/CDMA communications in fading channels," IEEE Commun. Lett., vol. 3, pp. 85-87, Apr. 1999.

[189] T. Ottosson and A. Svensson, "On schemes for multirate support in DS-CDMA systems," in Wireless Personal Communications. $\quad$ Norwell, MA: Kluwer, Mar. 1998, vol. 6, pp. 265-287.

[190] S. Ramakrishna and J. M. Holtzman, "A comparison between single code and multiple code transmission schemes in a CDMA system," in Proc. IEEE Vehicular Technology Conf. (VTC'98), 1998, pp. 791-795.

[191] M. Saquib and R. Yates, "Decorrelating detectors for a dual rate synchronous DS/CDMA channel," in Wireless Personal Communications. $\quad$ Norwell, MA: Kluwer, May 1999, vol. 9, pp. 197-216.

[192] A.-L. Johansson and A. Svensson, "Successive interference cancellation schemes in multi-rate DS/CDMA systems," Wireless Inform. Networks, pp. 265-279, 1996.

[193] S. Abeta, S. Sampei, and N. Morinaga, "Channel activation with adaptive coding rate and processing gain control for cellular DS/CDMA systems," in Proc. IEEE Vehicular Technology Conf. (VTC'96), 1996, pp. 1115-1119.

[194] M. Hashimoto, S. Sampei, and N. Morinaga, "Forward and reverse link capacity enhancement of DS/CDMA cellular system using channel activation and soft power control techniques," in Proc. 7th Personal, Indoor and Mobile Radio Communications (PIMRC) Conf., 1997, pp. 246-250.
[195] J. S. Blogh, P. Cherriman, and L. Hanzo, "Adaptive beamforming assisted, power controlled dynamic channel allocation for adaptive modulation," in Proc. IEEE Vehicular Technology Conf. (VTC'99 Fall), Amsterdam, The Netherlands, Sept. 19-22, 1999, pp. 2348-2352.

[196] J. M. Holtzman and D. J. Goodman, Eds., "Multimedia personal communication networks," in Wireless Communications. Norwell, MA: Kluwer, 1993, pp. 289-304.

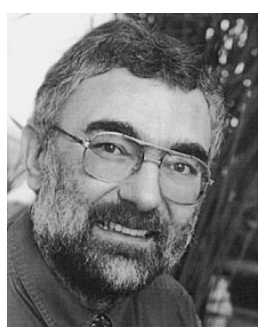

Lajos Hanzo (Senior Member, IEEE) received the M.S. degree in electronics and the Ph.D. degree from the Technical University of $\mathrm{Bu}$ dapest, Budapest, Hungary, in 1976 and 1983, respectively.

During his 24-year career in telecommunications, he has held various research and academic posts in Hungary, Germany, and the U.K. Since 1986, he has been with the Department of Electronics and Computer Science, University of Southampton, Southampton, U.K., and has been a consultant to Multiple Access Communications Ltd., U.K. Currently, he holds the chair in Telecommunications. He has coauthored five books on mobile radio communications, published about 300 research papers, organized and chaired conference sessions, presented overview lectures, and was awarded a number of distinctions. Currently, he is managing a research team, working on a range of research projects in the field of wireless multimedia communications under the auspices of the Engineering and Physical Sciences Research Council (EPSRC) U.K., the European IST Programme, and the Mobile Virtual Centre of Excellence (VCE).

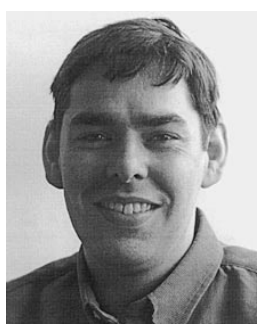

Peter Cherriman (Associate Member, IEEE) received the M.Eng. degree in information engineering from the University of Southampton, Southampton, U.K., in 1994, and the Ph.D. degree in mobile video networking from the same university.

Currently, he is working on projects for the Mobile Virtual Centre of Excellence, U.K. His current areas of research include robust video coding, microcellular radio systems, power control, dynamic channel allocation, and multiple

access protocols.

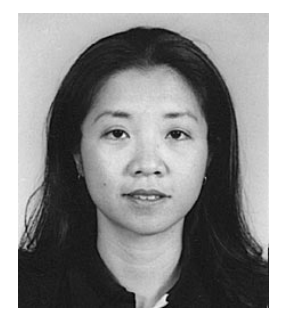

Ee-Lin Kuan received the B.Eng. degree in electronics engineering (First Class Honors) and the $\mathrm{Ph} . \mathrm{D}$. degree in mobile communications from the University of Southampton, Southampton, U.K.

She is currently with Multiple Access Communications Ltd., Southampton, U.K. Her current research interests are associated with mobile transceiver designs with emphasis on adaptive CDMA techniques. 